\title{
PI3K alpha and delta promote hematopoietic stem cell activation
}

\author{
Shayda Hemmati, ${ }^{1,2}$ Taneisha Sinclair,, ${ }^{1,2}$ Meng Tong, ${ }^{1,2}$ Boris Bartholdy, ${ }^{2}$ Rachel O. Okabe,,2 \\ Kristina Ames, ${ }^{1,2}$ Leanne Ostrodka, ${ }^{1,2}$ Tamanna Haque, ${ }^{2,3}$ Imit Kaur, ${ }^{1,2}$ Taylor S. Mills, ${ }^{4}$ \\ Anupriya Agarwal, ${ }^{5}$ Eric M. Pietras, ${ }^{4}$ Jean J. Zhao, ${ }^{6}$ Thomas M. Roberts, ${ }^{6}$ and Kira Gritsman ${ }^{1,2,3}$ \\ 'Department of Medicine and ${ }^{2}$ Department of Cell Biology, Albert Einstein College of Medicine, New York, New York, USA. \\ ${ }^{3}$ Department of Medical Oncology, Montefiore Hospital, New York, New York, USA. ${ }^{4}$ Division of Hematology, University of \\ Colorado Anschutz Medical Campus, Aurora, Colorado, USA. ${ }^{5}$ Knight Cancer Institute, Oregon Health Sciences University, \\ Portland, Oregon, USA, ${ }^{6}$ Department of Cancer Biology, Dana-Farber Cancer Institute, Boston, Massachusetts, USA.
}

\begin{abstract}
Many cytokines and chemokines that are important for hematopoiesis activate the PI3K signaling pathway. Because this pathway is frequently mutated and activated in cancer, PI3K inhibitors have been developed for the treatment of several malignancies and are now being tested in the clinic in combination with chemotherapy. However, the role of PI3K in adult hematopoietic stem cells (HSCs), particularly during hematopoietic stress, is still unclear. We previously showed that the individual PI3K catalytic isoforms $\mathrm{p} 110 \alpha$ and $\mathrm{p} 110 \beta$ have dispensable roles in HSC function, suggesting redundancy between PI3K isoforms in HSCs. We now demonstrate that simultaneous deletion of $\mathrm{p} 110 \alpha$ and $\mathrm{p} 110 \delta$ in double-knockout (DKO) HSCs uncovers their redundant requirement in $\mathrm{HSC}$ cycling after 5 -fluorouracil (5-FU) chemotherapy administration. In contrast, DKO HSCs were still able to exit quiescence in response to other stress stimuli, such as LPS. We found that DKO HSCs and progenitors had impaired sensing of inflammatory signals ex vivo, and that levels of IL-1 $\beta$ and MIC were higher in the bone marrow (BM) after LPS than after 5 -FU administration. Furthermore, exogenous in vivo administration of IL-1 $\beta$ could induce cell cycle entry of DKO HSCs. Our findings have clinical implications for the use of PI3K inhibitors in combination with chemotherapy.
\end{abstract}

Conflict of interest: The authors have declared that no conflict of interest exists.

Copyright: (c) 2019, American Society for Clinical Investigation.

Submitted: October 26, 2018

Accepted: May 17, 2019

Published: July 11, 2019.

Reference information: /CI Insight. 2019;4(13):e125832. https://doi. org/10.1172/jci.insight.125832

\section{Introduction}

Hematopoietic stem cells (HSCs), which are at the top of the hematopoietic hierarchy, exist mostly in a quiescent state. Under stress conditions such as infection, or after exposure to cytotoxic chemotherapy, HSCs become activated and enter the cell cycle, which drives emergency myelopoiesis and rapid regeneration of the hematopoietic system (1). These stimuli lead to the secretion of proinflammatory cytokines by a variety of immune cells, including myeloid cells and T cells, stromal cells in the BM microenvironment, and HSCs themselves (2). Furthermore, recent data suggest that HSCs can also directly respond to inflammatory signals, which leads to their activation, proliferation, and differentiation along the myeloid lineage $(3,4)$. Several different inflammatory signals are known to activate HSCs, including IFN- $\alpha$, IFN- $\gamma$, TLR ligands, LPS, IL- $1 \beta$, TNF- $\alpha$, and others $(1,5,6)$. Although many of these ligands activate similar downstream signaling pathways, the specific downstream effectors for these pathways in HSCs have not been delineated.

$\mathrm{PI} 3 \mathrm{~K}$ is a lipid and protein kinase that can be activated by receptor tyrosine kinases, cytokine receptors, chemokine G protein-coupled receptors, or oncogenes, including RAS. PI3K converts phosphatidylinositol 4,5-bisphosphate (PIP2) to phosphatidylinositol-3,4,5-triphosphate (PIP3), which leads to activation of pyruvate dehydrogenase kinase 1 (PDK1) and the serine/threonine kinase AKT (PKB) (7). AKT has many downstream effectors, which regulate the cell cycle, apoptosis, protein translation, autophagy, and metabolism. Most cytokines and RTK ligands signal through class IA PI3K.

Although several studies have also implicated the PI3K/AKT pathway downstream of IL-1 $\beta$ (8), TNF- $\alpha$ (9), or LPS/TLR signaling (10) in other cell types, the roles of PI3K/AKT in transducing inflammatory signals in hematopoietic stem and progenitor cells (HSPCs) are unknown. Furthermore, the specific roles of PI3K in HSC activation under stress are poorly understood. This is a critical question, as many 
kinase inhibitors and antibody-based therapeutics in clinical use can inhibit the PI3K/AKT pathway, and PI3K inhibitors are also used in cancer treatment (11). Because many patients undergoing such treatments also receive cytotoxic chemotherapy and are at increased risk for infection, it is important to understand how PI3K/AKT inhibition can affect the HSC stress response.

Class IA PI3Ks are composed of a p85 regulatory subunit and a p110 catalytic subunit. Hematopoietic cells express 3 different genes encoding 3 different isoforms of the class IA p110 subunit (Pik3ca, Pik3cb, $P i k 3 c d$ ), which encode $\mathrm{p} 110 \alpha, \mathrm{p} 110 \beta$, and $\mathrm{p} 110 \delta$, respectively (7). Because these 3 isoforms of p110 can all bind to p85, they can substitute for one another in some cellular contexts (7). Therefore, there is likely to be a high level of redundancy between the PI3K isoforms in HSCs. Using a conditional floxed-Pik3ca;Mx1-Cre mouse model, we previously demonstrated that $\mathrm{p} 110 \alpha$ is dispensable for HSC function, even after 5-fluorouracil (5-FU) administration (12). This was surprising, given that $\mathrm{p} 110 \alpha$ has been shown to be required for signaling in response to multiple growth factors in fibroblasts (13). We also observed normal peripheral blood counts in a floxed-Pik3cb;Mx1-Cre mouse model (14). These results support the hypothesis that PI3K isoforms play redundant roles in HSCs. Therefore, to examine the redundant roles of class I PI3K isoforms in HSCs at steady state and under stress conditions, we generated double-knockout (DKO) mice with germline deletion of $P i k 3 c d$ and conditional deletion of Pik3ca in the hematopoietic system. We uncovered significant redundancy between Pik3ca and Pik3cd in hematopoiesis, and also identified a role of these two PI3K isoforms in transducing inflammatory signals in HSPCs under stress conditions.

\section{Results}

p110 is not required for HSC repopulating function. To determine the most highly expressed class I PI3K isoforms in murine HSCs and early progenitors, we examined the expression of each of these isoforms in a previously published RNA sequencing data set (15). We found that, of the class IA isoforms, Pik3cd was expressed at the highest levels in HSCs and multipotential progenitors (MPPs) (Supplemental Figure 1A; supplemental material available online with this article; https://doi.org/10.1172/jci. insight.125832DS1). While Pik3cd germline homozygous knockout mice were previously described to have normal blood counts (16), a role for p110 $\delta$ in HSCs and early progenitors has not been reported. Consistent with previously published data (16), we confirmed that young homozygous Pik3cd germline knockout mice had normal blood counts (Supplemental Figure 1B). To determine whether p110 $\delta$ is required for HSC function, we performed competitive repopulation assays using whole BM from Pik3cd $d^{-/}\left(\mathrm{p} 110 \delta^{-/-}\right)$mice that were backcrossed into the C57BL/6 background for more than 9 generations, using C57BL/6 WT controls. We observed no significant differences in long-term multilineage repopulating ability between $\mathrm{p} 110 \delta^{-/-}$and WT BM, suggesting that $\mathrm{p} 110 \delta$ is not required for engraftment or multilineage reconstitution of the hematopoietic system (Supplemental Figure 1C). This finding further supports the hypothesis of redundancy between PI3K isoforms in HSCs.

$p 110 \alpha$ and $p 110 \delta$ play redundant roles in hematopoiesis. To determine whether $\mathrm{p} 110 \alpha$ and $\mathrm{p} 110 \delta$ play redundant roles in hematopoiesis, we generated DKO mice with the genotype Pik3cd ${ }^{-1} ;$ Pik3ca-lox/ lox;Mx1-Cre. Mx1 is a pan-hematopoietic promoter that can be induced postnatally by injecting mice with the double-stranded RNA polyinosinic:polycytidylic acid (pIpC). After 2 i.p. injections of pIpC at 4 weeks of age to excise Pik3ca, we observed significant leukopenia and anemia in DKO mice, while platelet counts were unchanged (Figure 1A). We observed a significant decrease in both neutrophil and lymphocyte counts in the peripheral blood of DKO mice (Figure 1B). DKO mice also had significantly decreased BM cellularity and decreased spleen weight (Figure 1C). Consistent with this observation, histological analysis revealed a hypocellular BM (Supplemental Figure 2A). While spleen weight was significantly decreased (Figure 1C), splenic architecture appeared intact in both $\mathrm{p} 110 \delta^{-{ }^{-}}$and DKO spleens (Supplemental Figure 2B). We observed no significant differences in the proportions of mature myeloid or B cells in the BM of DKO mice by flow cytometry, while the frequency of Thy $1.2^{+} \mathrm{T}$ cells was increased in the $\mathrm{p} 110 \delta^{-/-}$group (Supplemental Figure 2C). However, we did observe a significant decrease in the frequency of $\mathrm{B} 220^{+} \mathrm{B}$ cells in DKO and $\mathrm{p} 110 \delta^{-/-}$spleens, with a relative increase in the frequency of Mac1 $1^{\text {hi }} \mathrm{Gr}-1^{\text {hi }}$ myeloid cells and Thy $1.2^{+} \mathrm{T}$ cells in DKO spleens (Supplemental Figure 2D).

When we performed multiparameter flow cytometric analysis on the BM of DKO mice, p110 $\delta^{-/}$littermates, and WT;Mx1-Cre controls based on an established protocol (17), we observed no significant difference in the absolute numbers of phenotypically defined HSCs ( $\mathrm{Lin}^{-} \mathrm{c}-\mathrm{Kit}^{+} \mathrm{Sca}-$ $1^{+} \mathrm{Flk} 2^{-} \mathrm{CD} 150^{+} \mathrm{CD} 48^{-}$), short-term HSCs (ST-HSCs: Lin $^{-} \mathrm{c}-\mathrm{Kit}^{+} \mathrm{Sca}-1^{+} \mathrm{Flk2}{ }^{-} \mathrm{CD} 150^{-} \mathrm{CD} 48^{-}$), MMP2s 
A

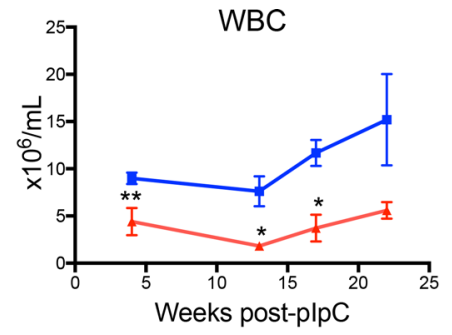

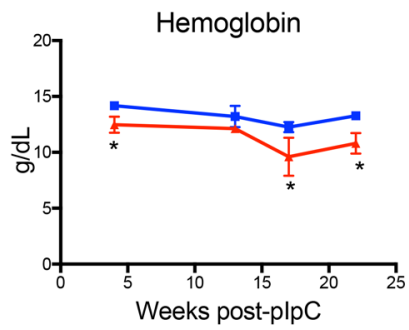

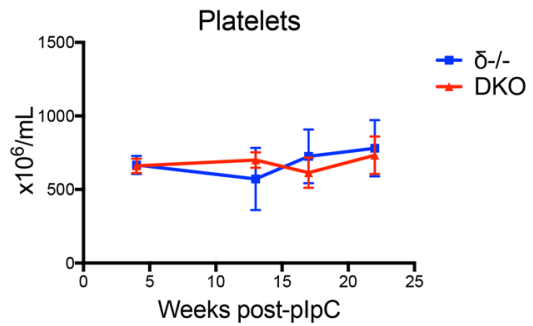

B
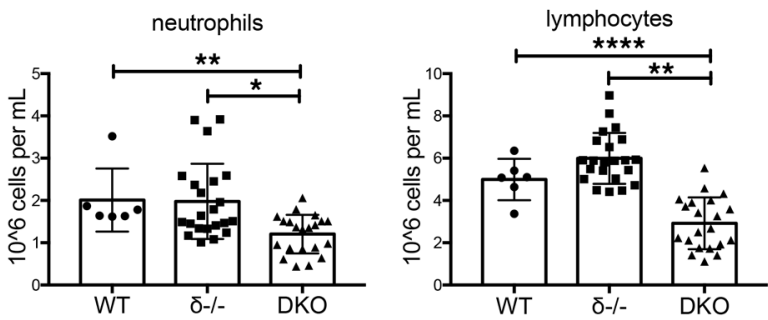

D

Lineage

low
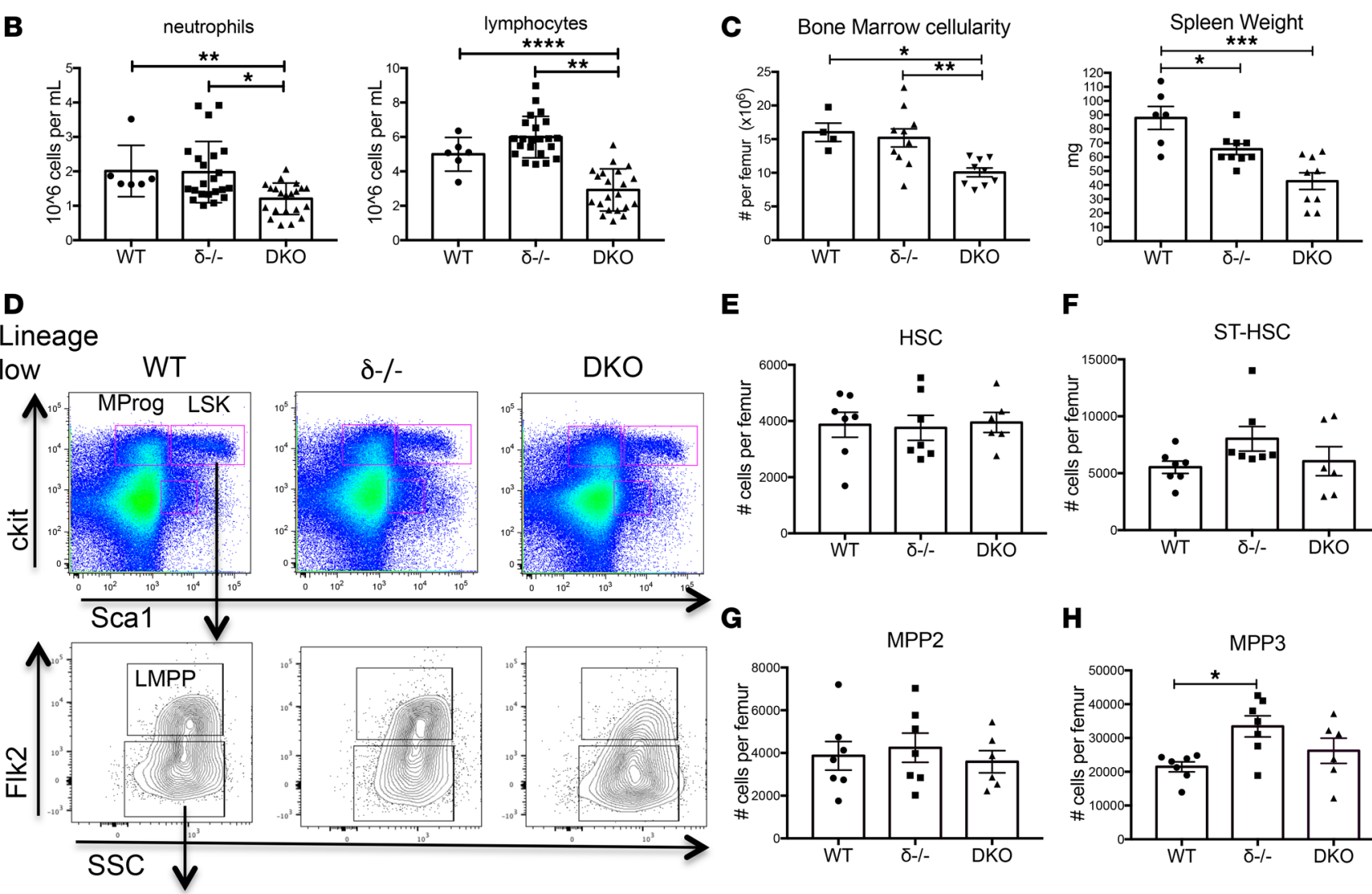

E
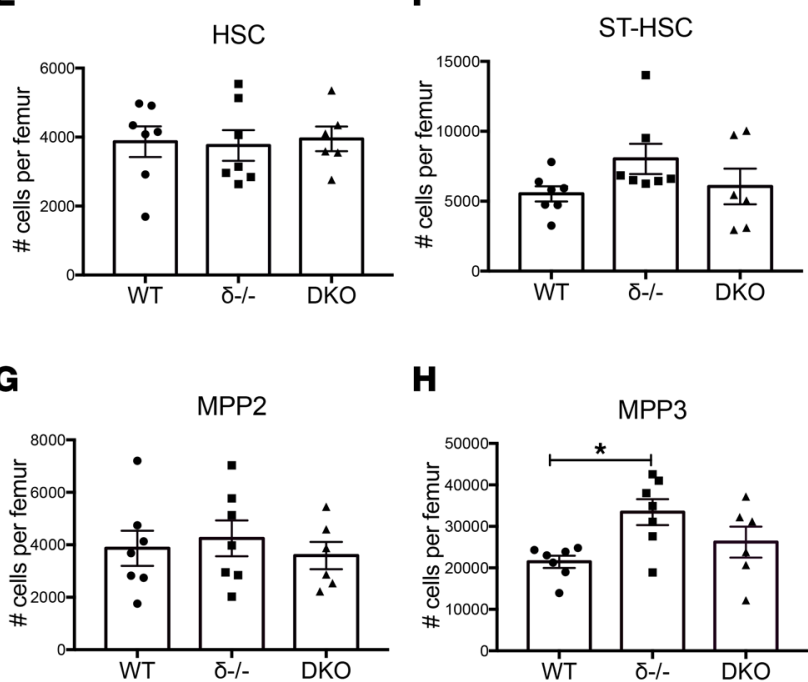

H
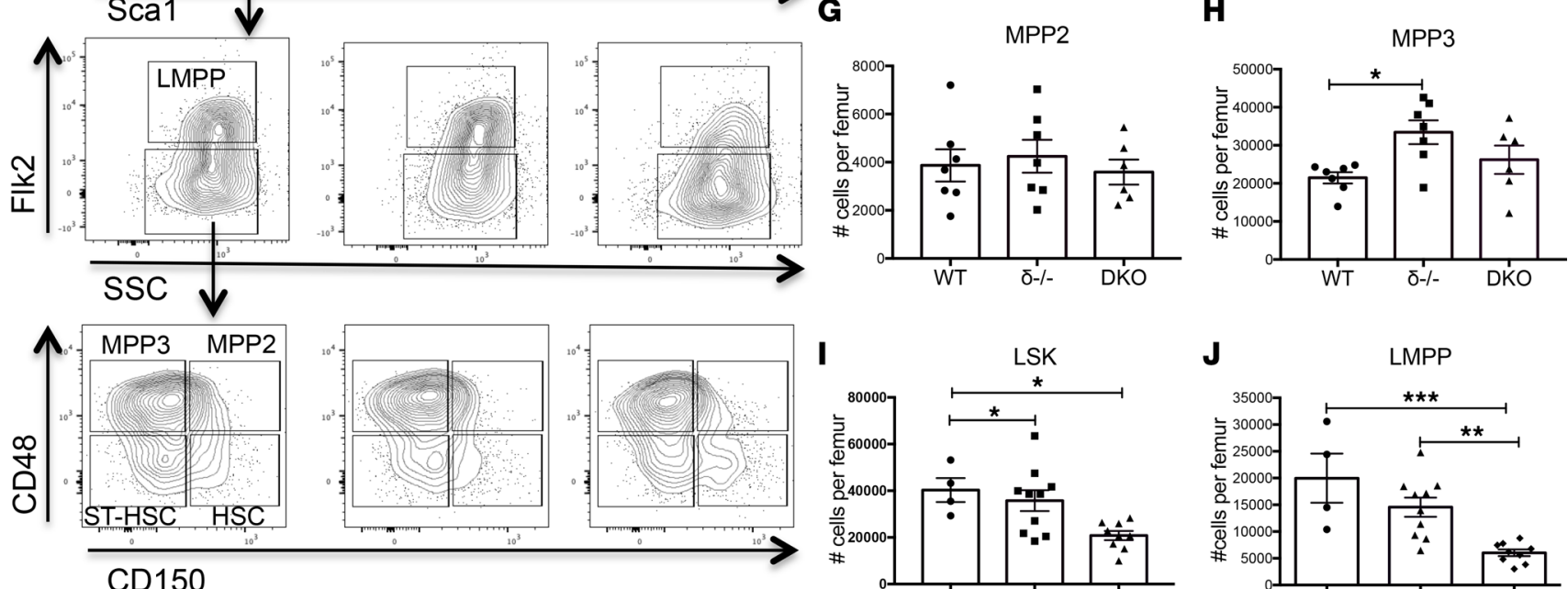

I
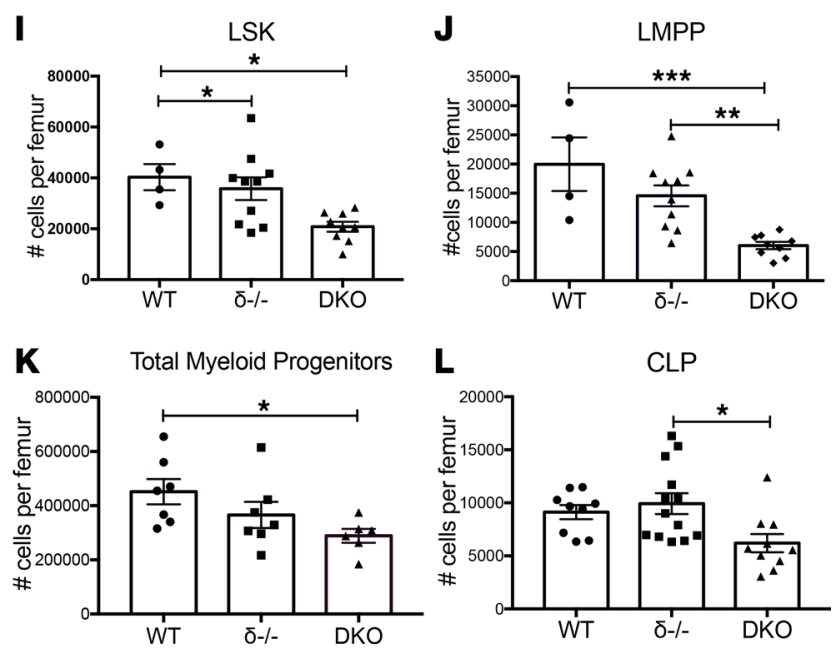

Figure 1. p110 $\alpha$ and p110 $\delta$ play redundant roles in hematopoiesis. (A) Serial peripheral blood counts of p110 $\alpha^{\text {lox/lox; }}$ p110 $\delta^{-1-} ; M \times 1-C r e ~ m i c e ~(D K O ; n=9)$ and $\mathrm{Cre}^{-}$p $110 \alpha^{\text {lox/lox}} ;$ p $110 \delta^{-1-}$ littermates $\left(\delta^{-1-} ; n=8\right)$ after 2 plpC injections. This experiment was performed 3 times. At each time point, unpaired 2 -tailed Student's $t$ test was used to compare the groups. (B) Peripheral blood absolute neutrophil and lymphocyte counts of DKO $(n=21), \delta^{-/-}(n=21)$, and WT;Mx1-Cre (WT, $n=6$ ) mice at 4 weeks after $2 \mathrm{plpC}$ injections. Combined data from multiple experiments are shown. (C) BM cellularity and spleen weights of DKO ( $n$ $=9), \delta^{-1-}(n=9)$, and age-matched WT C57BL/6 mice $(n=6)$ sacrificed at 4 weeks after plpC injection. (D) Representative flow cytometry plots of WT, $\delta^{-I_{-}}$ or DKO BM cells stained with HSC and progenitor markers. The gating strategy is shown for: Lin ${ }^{10} \mathrm{Sca}-\mathrm{h}^{\mathrm{hi}} \mathrm{C}-\mathrm{Kit}^{\mathrm{th}}$ (LSK), mixed myeloid progenitors (MProg), 
lymphoid-primed multipotent progenitors (LMPP), HSCs, short-term HSCs (ST-HSC), and multipotent progenitors 2 and 3 (MPP2 and MPP3). Absolute

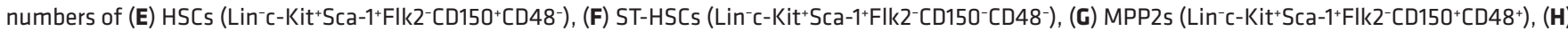

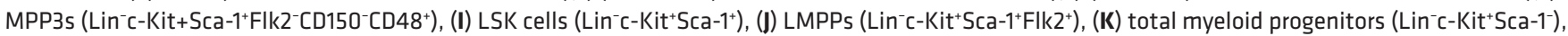
and (L) CLPs (Lin ${ }^{-}-\mathrm{Kit}^{\text {mid }} \mathrm{Sca}-\mathrm{1}^{\text {mid }} \mathrm{L}-7 \mathrm{R}^{+} \mathrm{Flk2} 2^{+}$) are shown. Combined data from 2 independent experiments are shown. This experiment was performed more than 3 times, with similar results. For the experiments shown in B-L, ANOVA with the Tukey's multiple-comparisons test was used. ${ }^{*} P<0.05,{ }^{* *} P<0.01$, ${ }^{* * *} P<0.001,{ }^{* * *} P<0.0001$. Data represent mean \pm SEM.

( Lin $\left.^{-} \mathrm{c}-\mathrm{Kit}^{+} \mathrm{Sca}-1^{+} \mathrm{Flk2}{ }^{-} \mathrm{CD} 150^{+} \mathrm{CD} 48^{+}\right)$, or MPP3s (Lin-c-Kit+Sca-1 ${ }^{+} \mathrm{Flk} 2^{-} \mathrm{CD} 150^{-} \mathrm{CD} 48^{+}$) between WT and DKO groups (Figure 1, D-H). However, we observed a significant decrease in the absolute number of LSK cells ( Lin $^{-} \mathrm{Sca}-1^{+} \mathrm{c}-\mathrm{Kit}^{+} ;$Figure 1I), which contain HSCs and MPPs, in lymphoid-primed MPPs

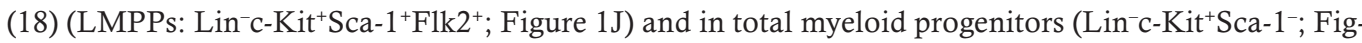
ure $1 \mathrm{~K}$ ) between the WT and DKO groups. We also observed a decrease in common lymphoid progen-

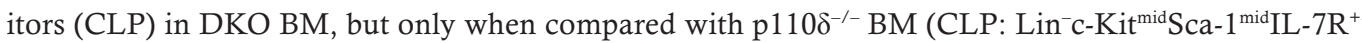
Flk2 ${ }^{+}$; Figure 1L and Supplemental Figure 3A). To determine which stage of B cell differentiation in the BM is most affected in DKO mice, we performed Hardy fraction analysis using flow cytometry $(19,20)$ (Supplemental Figure 3B). Interestingly, we observed a significant decrease in the absolute numbers of multiple B cell progenitor populations in the BM, including the pre-pro B cell population (Supplemental Figure $3 \mathrm{~B}$ ). These results suggest that $\mathrm{p} 110 \alpha$ and $\mathrm{p} 110 \delta$ play redundant roles at the earliest stages of $\mathrm{B}$ cell specification. In contrast, we did not observe any significant differences in the absolute number of common myeloid progenitors (CMPs), granulocyte-macrophage progenitors (GMPs), or megakaryocyte-erythroid progenitors (MEPs) in the BM (Supplemental Figure 4A).

However, methylcellulose plating of whole BM revealed a significant decrease in the numbers of total myeloid colonies produced by either $\mathrm{p} 110 \delta^{-/-}$or DKO BM cells (Supplemental Figure 4B), granulocyte-macrophage colonies (Supplemental Figure 4C), and monocyte colonies (Supplemental Figure 4D), with a trend toward a decrease in granulocyte colonies (Supplemental Figure 4E), while the number of granulocyte erythroid myelomonocytic (GEMM) colonies was unchanged in DKO BM from the WT control (Supplemental Figure 4F). Together, these data suggest that $\mathrm{p} 110 \alpha$ and p110 play redundant roles in hematopoiesis, particularly in specification of the B cell lineage. In addition, p1108 may also be important for the proliferation and/or differentiation of HSCs and progenitors.

$p 110 \alpha$ and $p 110 \delta$ are required for multilineage reconstitution. To examine the effects of $\mathrm{p} 110 \alpha$ and $\mathrm{p} 110 \delta$ deletion on HSC function, and to determine whether the effects of $p 110 \alpha$ and $p 110 \delta$ deletion are cell

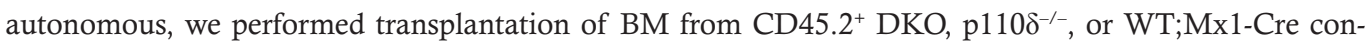
trols into lethally irradiated CD $45.1^{+}$B6.SJL recipient mice. We induced excision of $\mathrm{p} 110 \alpha$ at 4 weeks after transplantation only in recipient mice with a single $\mathrm{pIpC}$ injection. We confirmed excision of Pik3ca in the transplant recipients after one pIpC injection by excision PCR (Supplemental Figure 4G). We observed significant leukopenia in the transplant recipients in the DKO group, suggesting that this phenotype is cell autonomous. However, the transplant recipients did not develop anemia (Figure 2A). All transplant recipients survived for the entire 16-week observation period, suggesting that $\mathrm{p} 110 \alpha$ and $\mathrm{p} 110 \delta$ are not required for HSC and progenitor maintenance in the noncompetitive setting.

To examine HSC repopulating function under more stringent conditions, we performed competitive repopulation experiments, in which we transplanted BM cells from DKO or $\mathrm{p} 110 \delta^{-/-}$control CD $45.2^{+}$ donors at a 1:1 ratio with WT competitor BM cells from CD45.2 $2^{+}$CD $45.1^{+}$C57BL/6;B6.SJL mice into lethally irradiated $\mathrm{CD} 45.1^{+} \mathrm{B} 6 . \mathrm{SJL}$ recipients. After confirming engraftment, we injected the recipients with $\mathrm{pIpC}$ at 4 weeks after transplantation to excise Pik3ca. We found that deletion of both $\mathrm{p} 110 \alpha$ and $\mathrm{p} 110 \delta$ led to a significant and sustained decrease in total peripheral blood donor chimerism in the DKO group, compared with the $\mathrm{p} 110 \delta^{-/-}$group (Figure 2B). However, examination of individual peripheral blood lineages revealed the most profound decrease in donor chimerism in the $\mathrm{B} 220^{+} \mathrm{B}$ cell population, while the effects on the myeloid $\left(\mathrm{Macl}^{+} \mathrm{Grl}^{+}\right)$and $\mathrm{T}$ cell $\left(\right.$ Thy $\left.1.2^{+}\right)$lineages were less pronounced (Figure 2B). The effects on the B cell lineage were not surprising, since the roles of $\mathrm{p} 110 \delta$ in B cell receptor signaling and redundant roles of $\mathrm{p} 110 \alpha$ and $\mathrm{p} 110 \delta$ in B cell development in the BM have been described previously (16, 21). While there was a significant decrease in total BM donor chimerism in the DKO group, we did not observe a significant decrease in chimerism in the Flk $2^{-} \mathrm{CD} 150^{+}$LSK compartment, which contains HSCs (Figure 2C). However, donor chimerism was decreased in both Flk2- MPPs and Flk2 ${ }^{+}$LMPPs, as well as in CLPs (Figure 2C). We obtained similar results when we performed competitive repopulation experiments 
A

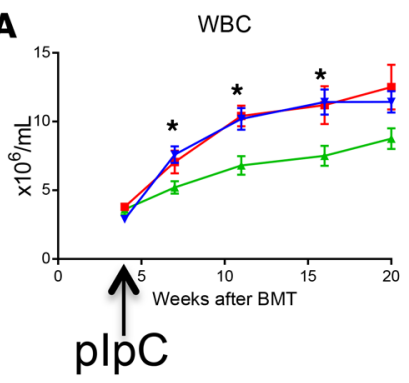

Hemoglobin

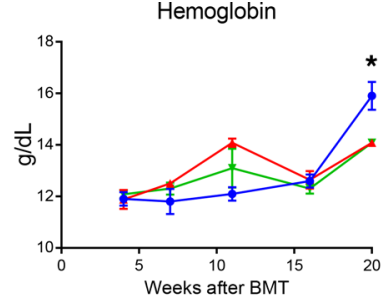

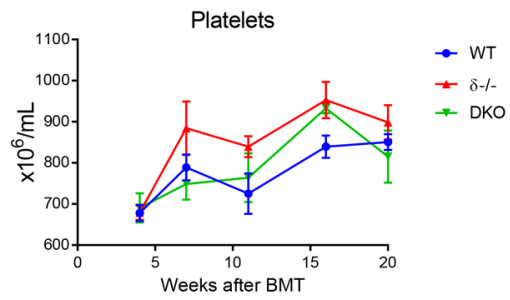

\section{B}
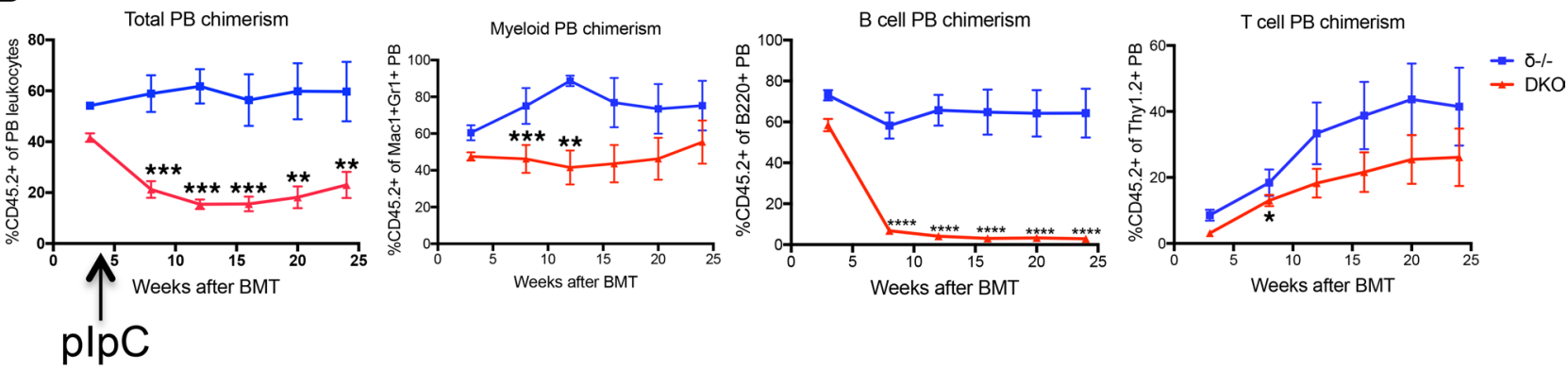

C
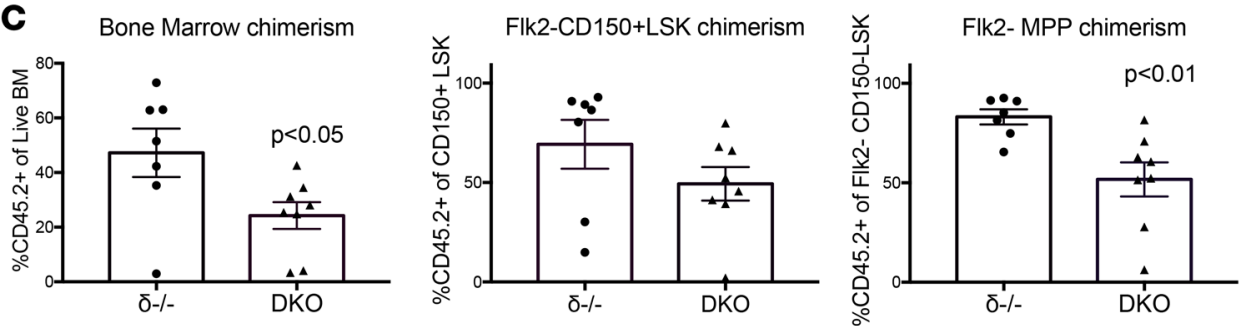

LMPP chimerism

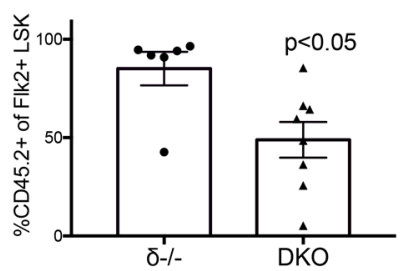

CLP chimerism

Myeloid Progenitor chimerism
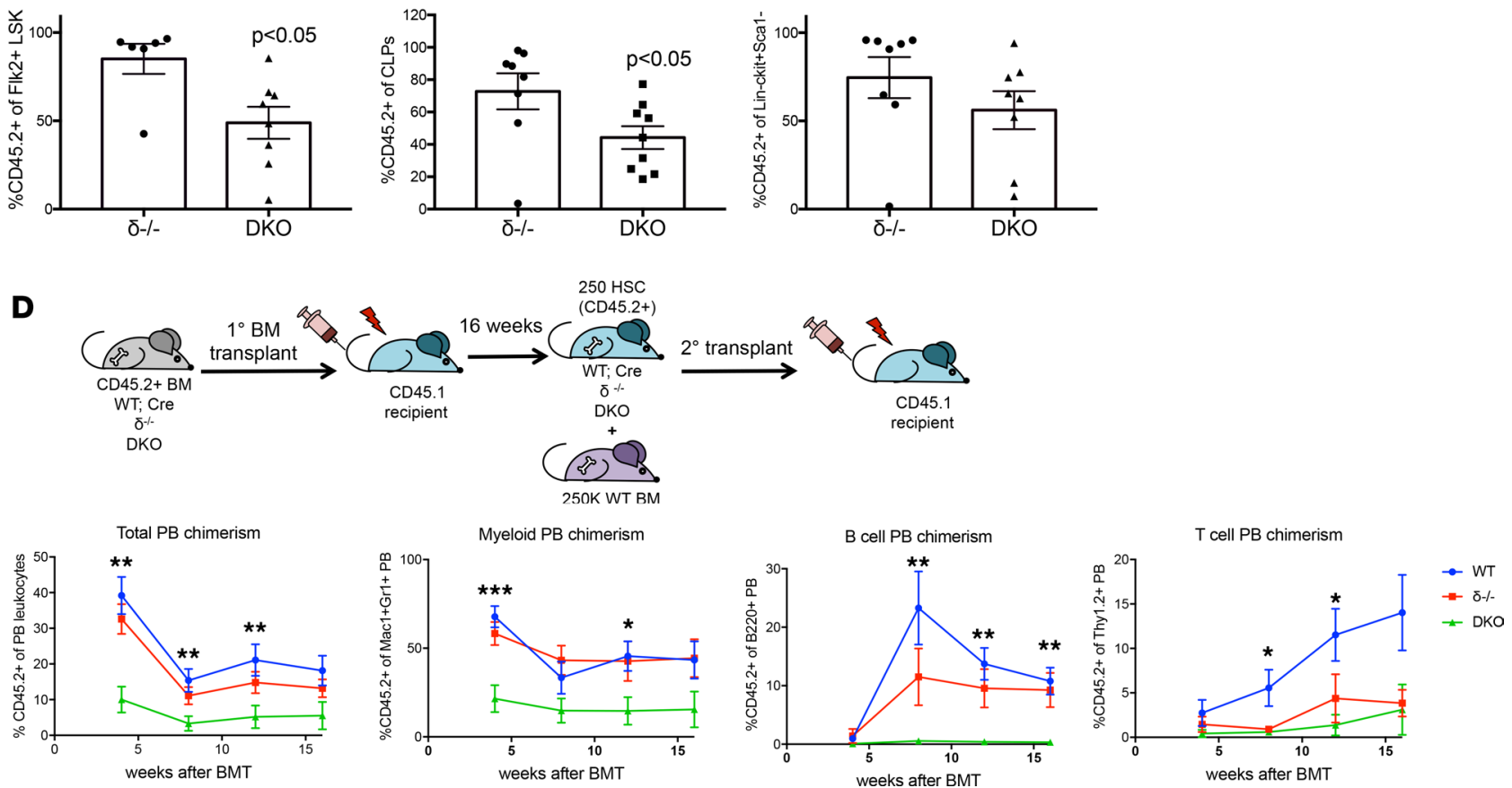

Figure 2. p110 $\alpha$ and $p 110 \delta$ play redundant roles in hematopoietic reconstitution. (A) Noncompetitive transplantation of $1 \times 10^{6} B M$ cells from p110 $\alpha^{\text {lox/lox- }}$ ; $110 \delta^{-1-} ; \mathrm{Mx1}$-Cre (DKO; $n=7$ ) donors, Cre- littermates $\left(\delta^{-1-} ; n=6\right)$, or age-matched WT;Mx1-Cre controls (WT; $n=7$ ) into 6- to 8-week-old female lethally irradiated B6.SJL recipients. Recipients were injected with plpC once at 4 weeks after transplantation. Serial peripheral blood counts of transplant recipients over time are shown from a representative experiment. This experiment was performed 3 times, with similar results. At each time point, ANOVA with the Tukey's multiple-comparisons test was used. (B) Competitive repopulation of DKO BM or $\delta^{-1-}$ (CD45.2+) BM mixed at a 1:1 ratio with CD45.2 $/$ CD45.1 WT BM from C57 BL6/B6.SJL F hybrids and transplanted into lethally irradiated B6.SJL (CD45.1 $1^{+}$) recipients $(n=10$ per group). 
The percentage of CD45.2 single-positive cells indicates the percent donor chimerism in each cell population. Representative data are shown from one of 2 experiments. At each time point, unpaired 2-tailed Student's $t$ test was used to compare the groups. (C) Donor chimerism in BM cell populations at the endpoint of the competitive repopulation experiment ( 25 weeks after transplantation). Representative data from one of 2 experiments are shown. (D) Secondary transplantation of sorted HSCs from primary BMT recipients of WT;Cre, DKO, or $\delta^{-1-}$ BM together with 250,000 CD45.1+CD45.2+ WT competitor BM cells. Serial donor chimerism analysis on peripheral blood (PB) populations is shown for WT;Cre $(n=8), \delta^{-1-}(n=8)$, and DKO $(n=8)$ transplant recipients. For the experiments shown in $\mathbf{C}$ and $\mathbf{D}$, ANOVA with the Tukey's multiple-comparisons test was used at each time point. ${ }^{*} P<$ $0.05,{ }^{* *} P<0.01,{ }^{* *} P<0.001,{ }^{* * *} P<0.0001$. Data represent mean \pm SEM.

by transplanting BM from DKO or $P i k 3 c t^{-1}$ mice that had been pretreated with pIpC (Supplemental Figure 5, A and B), suggesting that $\mathrm{p} 110 \alpha$ and $\mathrm{p} 110 \delta$ are dispensable for HSC engraftment. These results suggest that $\mathrm{p} 110 \alpha$ and $\mathrm{p} 110 \delta$ are more important for B cell differentiation and proliferation than for multilineage repopulation. This also suggests that $\mathrm{p} 110 \alpha$ and $\mathrm{p} 110 \delta$ do not play a critical role in HSCs under these conditions, but may play important roles in specific progenitor populations.

To determine whether $\mathrm{p} 110 \alpha$ and $\mathrm{p} 110 \delta$ are required for HSC self-renewal and multilineage repopulation under conditions requiring HSC activation, we performed secondary transplantation of 250 sorted Lin $^{-}{ }^{-}-\mathrm{Kit}^{+} \mathrm{Sca}-1^{+} \mathrm{Flk} 2^{-} \mathrm{CD} 48^{-} \mathrm{CD} 150^{+} \mathrm{HSC}$ from primary noncompetitive transplant recipient mice in combination with 250,000 WT competitor BM cells from CD45.2+/CD45.1 $1^{+}$C57BL/6;B6.SJL hybrids into lethally irradiated CD45.1 $1^{+}$B6.SJL recipients. Interestingly, we observed a significant decrease in the reconstitution of both myeloid and lymphoid lineages in the secondary transplant recipients (Figure 2D). Analysis of the BM of secondary transplant recipients revealed a significant decrease in total BM donor chimerism in the DKO group, and a trend toward decreased HSC donor chimerism (Supplemental Figure 5, C and D). We also observed a significant decrease in donor chimerism in the ST-HSCs, MPP3s, MPP4s, CLPs, and all myeloid progenitor populations in the DKO group (Supplemental Figure 5, E-L). These data reveal that DKO HSCs are defective in contributing to multiple mature lineages under stress, including the myeloid lineage. This suggests that $\mathrm{p} 110 \alpha$ and $\mathrm{p} 110 \delta$ are important for the regulation of HSC function during hematopoietic stress.

Deletion of $p 110 \alpha$ and $p 110 \delta$ in HSCs affects gene expression programs associated with cell cycling and inflammatory signaling. To understand the molecular mechanisms of how $\mathrm{p} 110 \alpha$ and $\mathrm{p} 110 \delta$ regulate the HSC and progenitor stress response, we examined the effects of $\mathrm{p} 110 \alpha$ and $\mathrm{p} 110 \delta$ deletion on gene expression in HSCs and LMPPs. We sorted HSCs ( $\left.\mathrm{Lin}^{-} \mathrm{C}-\mathrm{Kit}^{+} \mathrm{Flk} 2^{-} \mathrm{CD} 150^{+} \mathrm{CD} 48^{-}\right)$and $\mathrm{LMPPs}\left(\mathrm{Lin}^{-} \mathrm{C}-\mathrm{Kit}^{+} \mathrm{Flk} 2^{+} \mathrm{CD} 150^{-}\right.$ $\mathrm{CD}_{4}{ }^{+}$) from pooled BMT recipients, and performed RNA microarray analysis in triplicate using the Affymetrix Mouse Transcriptome Assay 1.0 kit (Figure 3A). We observed significantly altered gene expression in DKO HSCs compared with WT HSCs (Figure 3, B and C, and Supplemental Table 1). Significantly altered expression of some genes ( $>2$-fold change, $P<0.05$ ) between $\mathrm{p} 110 \delta^{-/}$HSCs and WT HSCs, or between p110 $\alpha^{-/-}$HSCs and WT HSCs, was also observed (Figure 3C and Supplemental Figure 6A). Interestingly, we did not observe any significant increase in expression of Pik3ca in $\mathrm{p} 110 \delta^{-/-} \mathrm{HSCs}$ or of Pik3cd in $\mathrm{p} 110 \alpha^{-/-}$HSCs or LMPPs in this dataset, suggesting that compensation between these isoforms at the transcriptional level is unlikely (Supplemental Tables 1 and 2). Furthermore, there were few overlapping dysregulated genes among the 3 comparisons, suggesting distinct roles for each isoform in regulating gene expression within this HSC-enriched population (Figure 3C). It is possible that these distinct gene expression patterns reflect enrichment for specific cell populations among the different genotypes, even within the purified $\mathrm{Lin}^{-} \mathrm{C}-\mathrm{Kit}^{+} \mathrm{Flk} 2^{-} \mathrm{CD} 150^{+} \mathrm{CD} 48^{-}$bulk cell population. Consistent with this, we observed by gene set enrichment analysis (GSEA) using the Molecular Signatures Database (MSigDB; Broad Institute) positive enrichment of a previously published mouse long-term HSC-associated gene set in $\mathrm{p} 110 \delta^{-/-} \mathrm{HSCs}$ compared with WT HSCs (Supplemental Figure 6A) (17). Similarly, we observed positive enrichment of another HSC-associated gene set in DKO HSCs, when compared with either $\mathrm{p} 110 \alpha^{-/-}$or $\mathrm{p} 110 \delta^{-/-}$HSCs (Supplemental Figure 6, B and C). Furthermore, we saw negative enrichment of a lymphoid stem cell signature in DKO HSCs compared with $\mathrm{p} 110 \delta^{-/-}$HSCs (Supplemental Figure 6D). These results suggest that

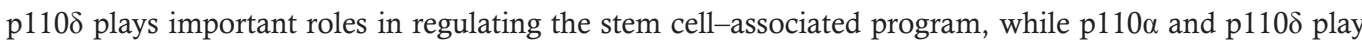
redundant roles in early B lineage specification.

GSEA also revealed that the most significantly negatively enriched gene sets in DKO HSCs compared with WT HSCs were those associated with cell cycling, such as HALLMARK_G2M_CHECKPOINT and HALLMARK_E2F_TARGETS (Figure 3D and Supplemental Table 3). As expected, we observed negative enrichment of gene sets associated with PI3K/mTOR signaling, which validates our microarray 
A

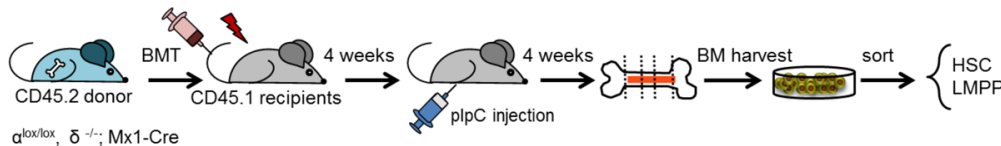

$\delta^{-1-} \mathrm{M} \times 1-\mathrm{Cr}$

$a^{10 x / 10 x}, \delta$

$\mathrm{a}^{\text {loxllox }} ; \mathrm{Mx} 1-\mathrm{Cre}$

$a^{\text {lox/lox }}$

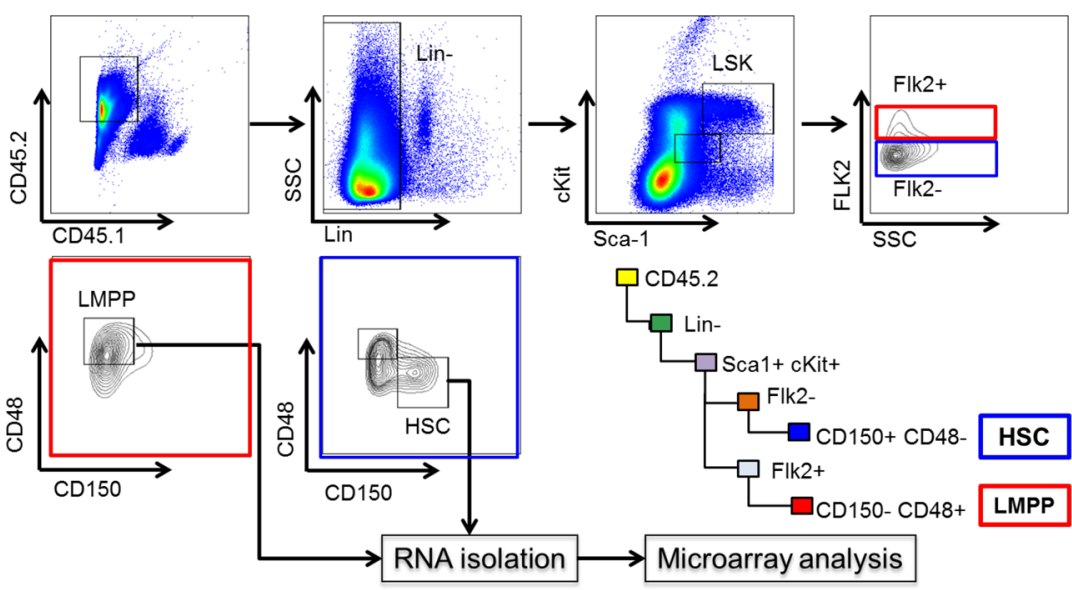

B DKO WT

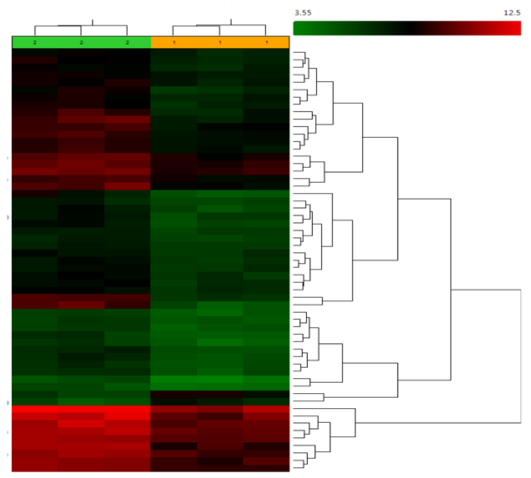

C

Upregulated in HSC

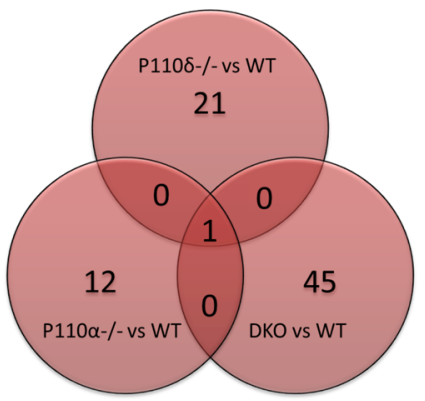

Downregulated in HSC

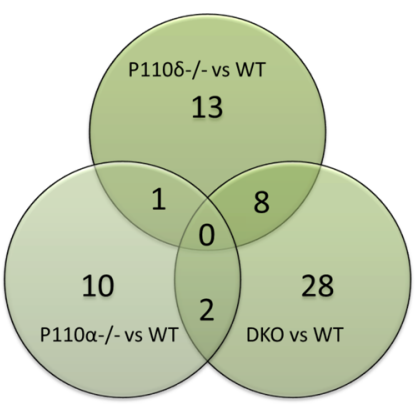

D
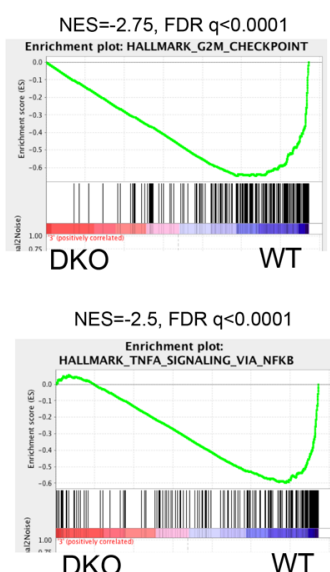

$\mathrm{NES}=-2.52$, FDR $q<0.0001$

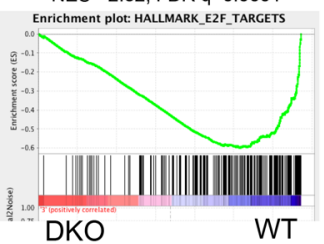

NES $=-1.67$, FDR $q=0.004$

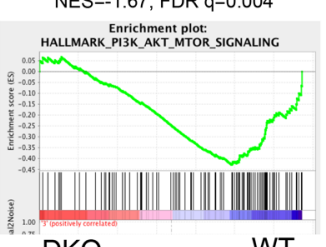

E

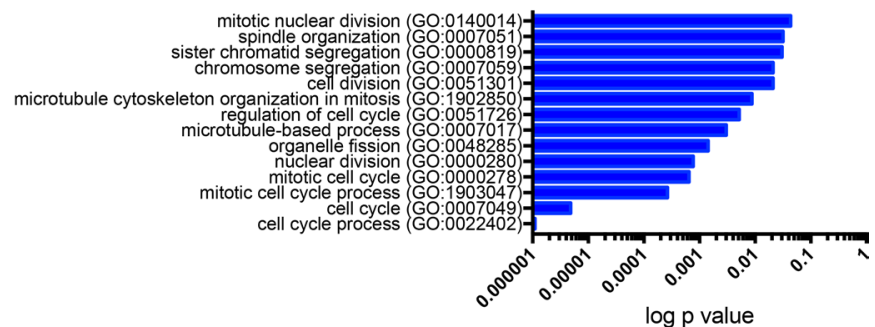

$\log p$ value

Figure 3. Downregulation of gene sets associated with cell cycle progression and inflammatory signaling in DKO HSCs. (A) Experimental design for BM transplantation and sorting of HSCs and LMPPs for microarray analysis. BM cells of each genotype (CD45.2+) were transplanted from one donor into 7 WT recipients (CD45.1+) each, and the harvested BM from each set of 7 transplanted mice was pooled for sorting and RNA synthesis. Transplantation was performed 3 times for each donor genotype, resulting in a total of 3 replicates per genotype. (B) Heatmap of genes differentially expressed between DKO HSCs and WT HSCs. (C) Venn diagrams representing the total number of differentially upregulated or downregulated genes between HSCs of each genotype compared with WT HSCs ( $>2$-fold change with $P<0.05$ ). (D) Representative GSEA plots some of the gene sets with the highest negative enrichment scores (NES) when compared with our DKO versus WT HSC gene set. (E) Gene ontology (GO) analysis of significantly downregulated genes ( $>2$-fold, $P<0.05$ ) in DKO versus WT HSCs. 
A

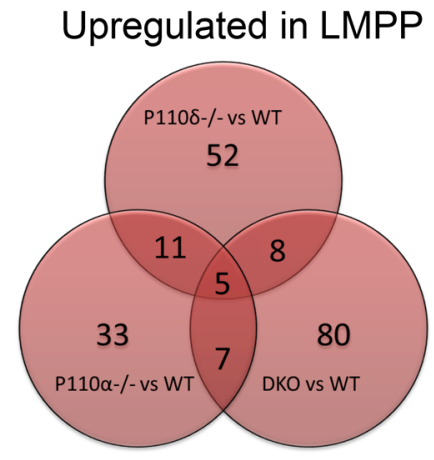

B

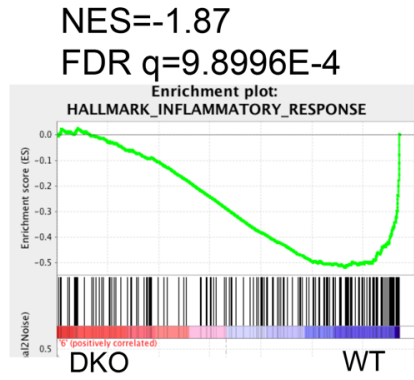

\section{Downregulated in LMPP}

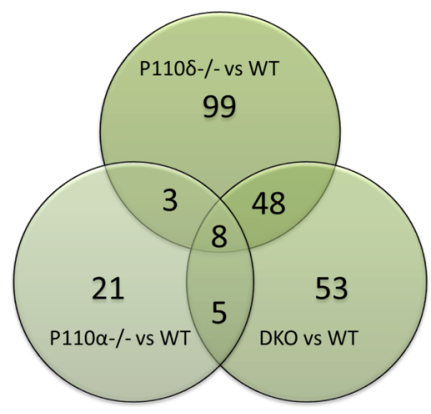

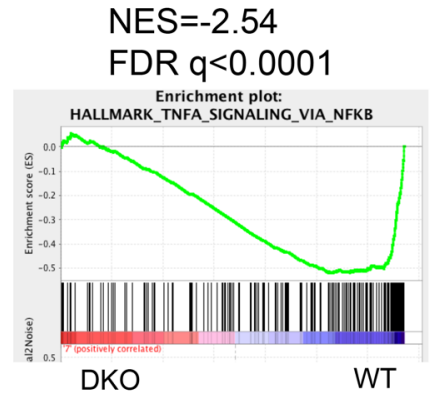

$\mathrm{NES}=-2.54$ FDR $\mathrm{q}<0.0001$ Enrichment plot:
HALLMARK_TNFA_SIGNALING_VIA_NFKB

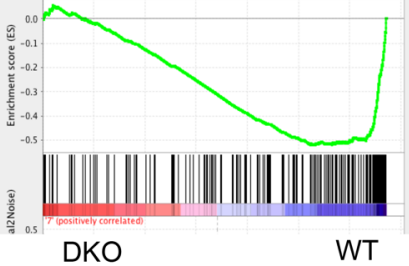

NES $=-1.72$

FDR $\mathrm{q}<0.0001$

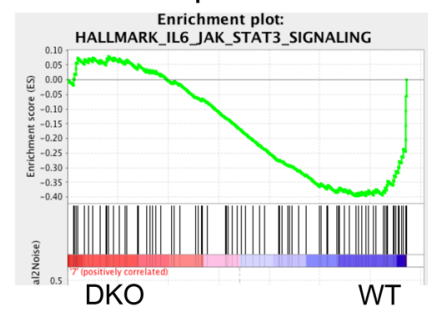

C

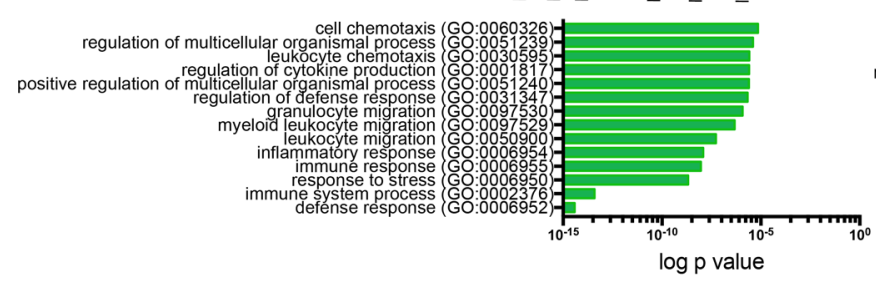

D

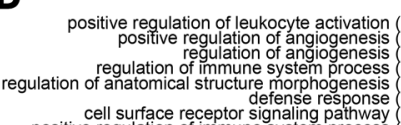
cell surface receptor defignaling response
posithway immune response
cellular response to stimulus
immune system proces 난?
DOWN_IN_DKO_LMPP_VS_WT_LMPP

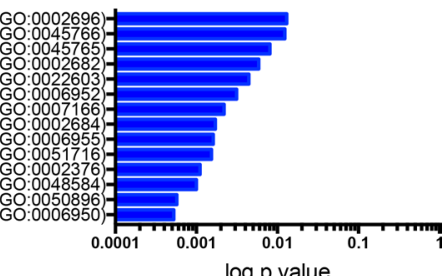

Figure 4. Downregulation of inflammatory signaling gene sets in DKO LMPPs. (A) Venn diagrams representing the total number of differentially upregulated or downregulated genes between LMPPs of each genotype compared with WT HSCs (>2-fold change with $P<0.05)$. (B) Representative GSEA plots of the gene sets with the lowest negative enrichment score (NES) when compared with our DKO versus WT LMPP gene set. Gene ontology (CO) analysis of significantly downregulated genes ( $>2$-fold, $P<0.05$ ) in (C) $p 110 \delta^{-1-}$ LMPPs versus WT LMPPs and (D) in DKO LMPPs versus WT LMPPs.

analysis results (Figure 3D and Supplemental Table 3). Interestingly, we also observed significant negative enrichment of gene sets associated with inflammatory signaling in DKO HSCs, such as HALLMARK_ TNFA_SIGNALING_VIA_NFKB, compared with both WT HSCs and p110 $\alpha$-KO HSCs (Figure 3D and Supplemental Table 3). In addition, we observed negative enrichment of several other signaling pathways that have been implicated in HSC self-renewal, including the TGF- $\beta$ pathway, and the WNT/ $\beta$-catenin pathway (Supplemental Table 3). Gene ontology enrichment analysis (Gene Ontology Consortium) of the genes that were downregulated greater than 2-fold in DKO HSCs compared with WT HSCs with $P<0.05$ also revealed significant enrichment of multiple gene sets associated with the cell cycle (Figure 3E). Furthermore, Ingenuity Pathway Analysis (IPA, QIAGEN) of genes that were dysregulated greater than 2-fold in DKO HSCs compared with WT HSCs with $P<0.05$ also revealed significant downregulation of several pathways associated with the cell cycle (Supplemental Figure 6E).

Interestingly, we also observed negative enrichment of both the HALLMARK_TNFA_SIGNAL-

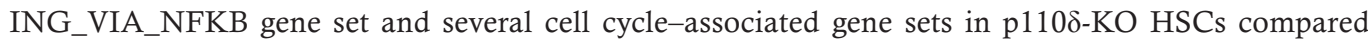
with WT HSCs (Supplemental Table 1). However, a direct comparison of gene expression in DKO HSCs compared with $\mathrm{p} 110 \delta^{-/-}$HSCs revealed more significant downregulation of cell cycle gene sets in DKO HSCs (Supplemental Table 3). In contrast, a comparison of DKO HSCs with p110 $\alpha^{-/-}$HSCs revealed more pronounced downregulation of the HALLMARK_TNFA_SIGNALING_VIA_NFKB module in DKO HSCs (Supplemental Table 3). We also observed significant positive enrichment of RAS pathway gene sets in $\mathrm{p} 110 \alpha^{-/-}, \mathrm{p} 110 \delta^{-/-}$, and DKO HSCs compared with WT HSCs, suggesting 
A

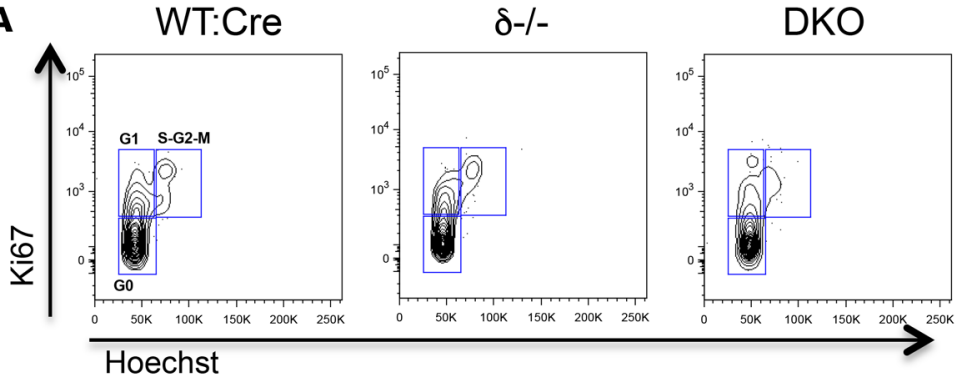

HSC

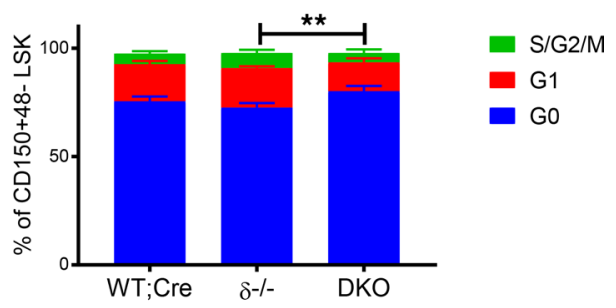

B
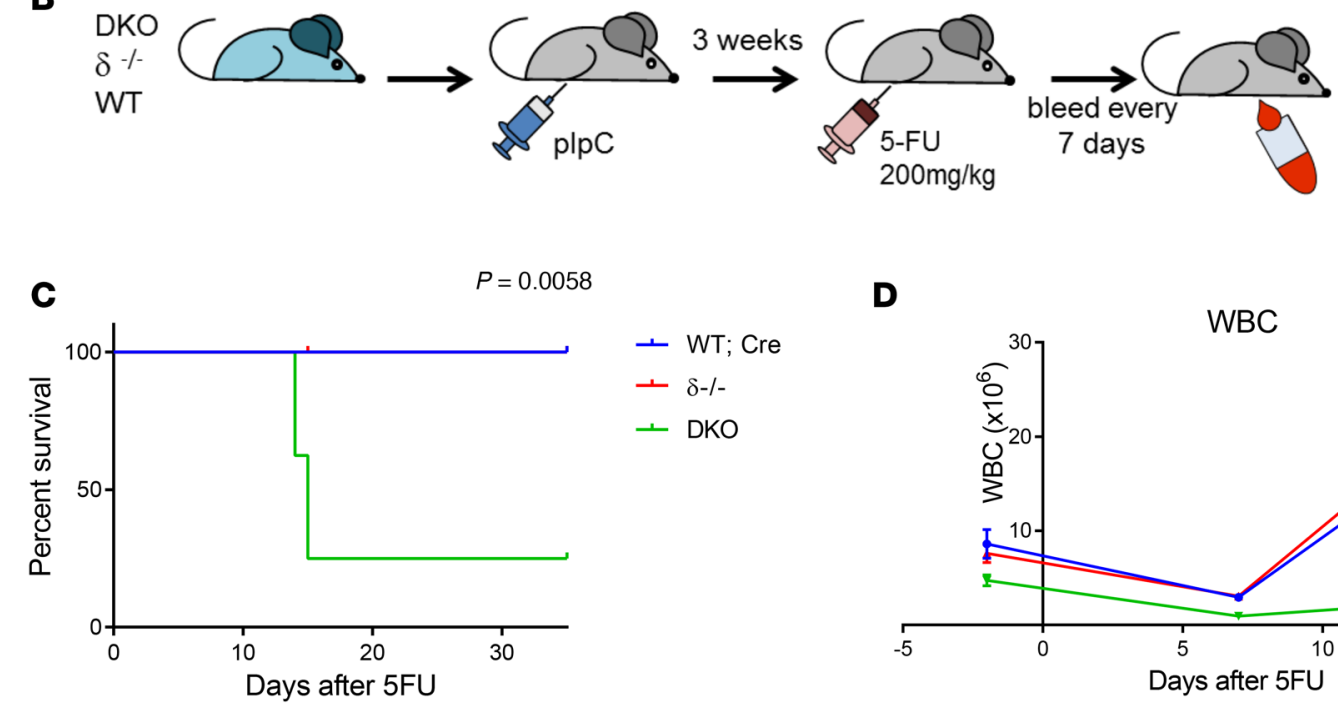

D

$$
\text { E }
$$

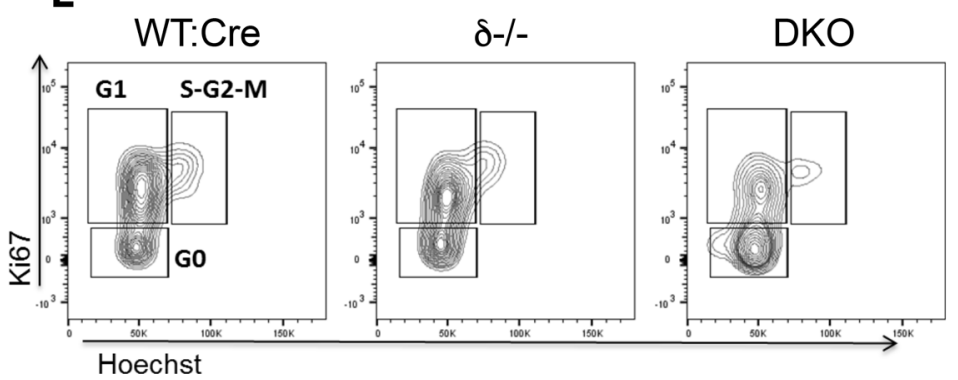

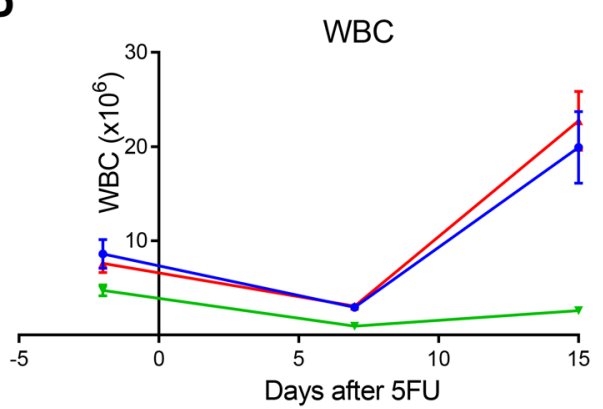

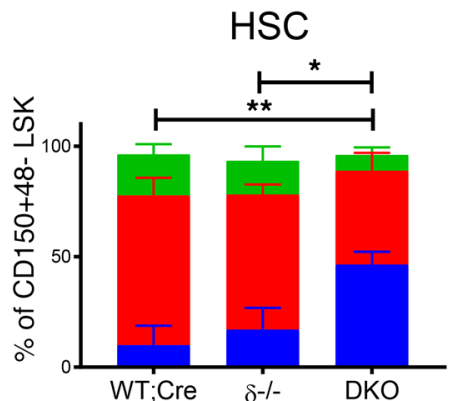

$\rightarrow$ WT;Cre

$\pm \delta-/-$

$\rightarrow$ DKO

Figure 5. DKO HSCs have an impaired cycling response to 5-fluorouracil injection. (A) Cell cycle analysis of HSCs (Linc-Kit ${ }^{+}$Sca-1+CD150+CD48- cells) from DKO ( $n=$ 4), $\delta^{-/-}(n=3)$, or WT; Cre $(n=3)$ BM at 3 weeks after 2 plpC injections. Representative Hoechst/Ki-67 HSC plots are shown from one of 3 independent experiments, with quantification of the frequency of $\mathrm{HSCs}$ found in $\mathrm{G}_{0}, \mathrm{G}_{1}$, or $\mathrm{S}-\mathrm{G}_{2}-\mathrm{M}$ phase of the cell cycle on the right. (B) Experimental schematic for 5 -fluorouracil (5-FU) experiments. (C) Kaplan-Meier survival curve of mice injected with plpC once, followed by a single injection of $200 \mathrm{mg} / \mathrm{kg} \mathrm{5-FU} \mathrm{(WT;Cre,} n=6$; p110 $\delta^{-1-}, n=5 ;$ DKO, $n=8$ ). Log-rank analysis was used to compare the groups. (D) Serial WBC counts of the mice shown in $\mathbf{C}$. Representative data from one of 3 experiments is shown. (E) Representative cell cycle analysis of HSCs in the BM of mice that were sacrificed at 7 days after 5-FU injection (WT;Cre, $n=3 ; \delta^{-1-}, n=3 ;$ DKO, $n=3$ ). Representative flow cytometry plots from one of 3 experiments are shown. Quantification of the frequency of HSCs found in $G_{0}, G_{1}$, or $S-G_{2}-M$ phase of the cell cycle is shown on the right. For the experiments in $\mathbf{A}$ and $\mathbf{E}$, ANOVA with Tukey's multiple-comparisons test was used. ${ }^{*} P<0.05$, ${ }^{* *} P<0.01$. Data represent mean $\pm \mathrm{SEM}$.

that the RAS/MAPK pathway may compensate for PI3K deletion in HSCs (Supplemental Table 3). Together, these data suggest some overlap between the roles of p110 $\alpha$ and p110 in HSCs, and also imply a functional redundancy between these $2 \mathrm{PI} 3 \mathrm{~K}$ isoforms in regulating the cell cycle in HSCs.

We also observed significant dysregulation of gene expression in DKO, p110 $\alpha^{-/-}$, and $\mathrm{p} 110 \delta^{-/-}$LMPPs compared with WT;Mx1-Cre controls (Figure 4A and Supplemental Table 2). There was a surprisingly limited overlap between the upregulated gene sets of single-KO LMPPs and DKO LMPPs, though this could represent a difference in the progenitor subpopulations contained within the LMPP gate between the 
3 genotypes (Figure $1 \mathrm{~J}$ and Figure 4A). However, there was a high degree of overlap between the downregulated genes in $110 \delta-\mathrm{KO}$ and DKO LMPPs, suggesting that many of these expression changes are caused by the deletion of $\mathrm{p} 110 \delta$. GSEA revealed significant negative enrichment in multiple inflammatory gene sets in DKO LMPPs compared with WT LMPPs, including HALLMARK_INFLAMMATORY_ RESPONSE, HALLMARK_TNFA_SIGNALING_VIA_NFKB, and HALLMARK_IL6_JAK_STAT3_ SIGNALING (Figure 4B and Supplemental Table 4). We also observed negative enrichment of similar gene sets in p1108-KO LMPPs (Supplemental Table 4). Gene Ontology analysis of the gene set downregulated greater than 2-fold $(P<0.05)$ in $110 \delta^{-/}$LMPPs or DKO LMPPs revealed enrichment for multiple inflammatory and stress response modules (Figure 4, C and D). Consistent with this finding, IPA of these downregulated gene sets confirmed the downregulation of several inflammatory signaling pathways in both p1108-KO and DKO LMPPs (Supplemental Figure 6F and Supplemental Tables 5 and 6). These data suggest that $\mathrm{p} 110 \delta$ could be important in the response of both HSCs and LMPPs to inflammatory signals.

$p 110 \alpha$ and $p 110 \delta$ are essential for hematopoietic recovery after 5-FU. Given our findings that $\mathrm{p} 110 \alpha$ and $\mathrm{p} 110 \delta$ appear to be more important in regulating HSC function under stress, we wondered whether the defect in cell cycle regulation we observed would be accentuated under stress conditions. To address this question, we first performed cell cycle analysis using Hoechst 33342 and Ki-67 staining on p110 $\delta^{-1-}$ and DKO HSPCs at 4 weeks after a single pIpC injection to excise Pik3ca. Although most HSCs were quiescent at steady state, we observed a modest increase in the quiescence of DKO HSCs compared with p1108-KO and WT HSCs (Figure 5A). To examine whether the effects of $\mathrm{p} 110 \alpha$ and $\mathrm{p} 110 \delta$ deletion on HSC cycling may be secondary to an impaired response to hematopoietic stress, we analyzed the hematopoietic system in $\mathrm{p} 110 \delta^{-/-}$and DKO mice after 5-FU administration. A single injection of 5-FU initially causes pancytopenia in mice due to depletion of mature blood cells, followed by cell cycle entry of HSCs and progenitors in order to rapidly replenish the hematopoietic system (22). We treated DKO mice, $\mathrm{p} 110 \delta^{-/-}$littermates, and WT;Mx1-Cre control mice with a single 5-FU injection $(200 \mathrm{mg} / \mathrm{kg}$ ) at 3 weeks following a single $\mathrm{pIpC}$ injection. We followed blood counts in all groups every 7 days until count recovery. Strikingly, while all of the WT and $\mathrm{p} 110 \delta^{-/}$mice survived 5-FU administration, and recovered normal blood counts within 21 days, 6 of 8 DKO mice died within 15 days after 5-FU injection (Figure 5, B and C). DKO mice remained pancytopenic at 15 days after 5-FU injection, while both control groups recovered their counts within this time period (Figure 5D). To understand the etiology of this pancytopenia, we performed a separate experiment in which all mice were sacrificed at 7 days after a single 5-FU injection $(180 \mathrm{mg} / \mathrm{kg})$, and the cell cycle status of BM populations was analyzed. Strikingly, while WT;Mx1-Cre and $\mathrm{p} 110 \delta^{-/-}$HSCs were actively cycling at this time point, with only $8 \%-15 \%$ of the cells in $\mathrm{G}_{0}$, we observed a significant increase in the proportion of DKO HSCs that remained quiescent after 5-FU treatment, with a significant decrease in the proportion of cycling cells (Figure 5E). Because the expression of several HSC markers, such as c-Kit and Sca-1, changes after 5-FU administration, making the gating of HSCs challenging, we also examined the cell cycle profile in other BM cell populations. We observed the same trend toward increased quiescence in myeloid progenitors, $\mathrm{Lin}^{-} \mathrm{Sca}-1^{+} \mathrm{c}-\mathrm{Kit}^{+}$cells, and total $\mathrm{Lin}^{-}$cells in DKO mice (Supplemental Figure 7). This impaired ability to enter the cell cycle upon stress likely can explain the lack of hematopoietic recovery and poor survival after 5-FU administration in DKO mice.

p110 is required for the maximal response of HSPCS to IL-1 $\beta$ and TNF- $\alpha$. 5-FU has been reported to increase the levels of several inflammatory cytokines in the BM, including IL-1 $\alpha$ and IL-1 $\beta$, and IL-1R signaling is required for myeloid recovery after 5-FU (23). Given the downregulation of inflammatory signaling gene sets that we detected in $\mathrm{p} 110 \delta^{-/-}$and DKO HSCs and LMPPs, and the defect in cell cycle entry after 5-FU injection in DKO HSPCs, we wondered whether the observed defect in HSC activation and cell cycle entry after 5-FU was due to the impaired ability of HSPCs to transduce inflammatory signals. One of the most significantly downregulated inflammatory signaling pathways observed in our IPA analysis in DKO LMPPs was the p38 MAPK signaling pathway (Supplemental Figure 6F). Interestingly, p38 MAPK is activated in BM cells after 5-FU treatment and has been found to be essential for promoting cell cycle progression of HSCs during stress hematopoiesis (24). The inflammatory cytokines IL- $1 \beta$ and TNF- $\alpha$ stimulate signaling pathways through the cell surface receptors IL-1R and TNFR, respectively, which leads to the phosphorylation of p38 MAPK (reviewed in ref. 25). Furthermore, TNF- $\alpha$ is known to signal through the PI3K/AKT pathway in other cell types (9). Therefore, we harvested BM cells from DKO, p110 $\delta^{-/}$, and WT;Mx1-Cre mice at 3 weeks after pIpC treatment, stimulated lineage-negative cells ex vivo with IL- $1 \beta$ or TNF- $\alpha$, and then examined phosphorylation of p38 MAPK in HSPCs and myeloid progenitors by intracellular phospho-flow cytometry. We observed a decreased level of p38 MAPK phosphorylation in both $\mathrm{p} 110 \delta^{-/-}$and DKO HSPCs, both at baseline and after stimulation with either IL-1 $\beta$ or 
TNF- $\alpha$ (Figure 6, A-D). To confirm the impaired response of $\mathrm{p} 110 \delta^{-/-}$and DKO cells to these inflammatory signals, we also performed Western blotting on c-Kit-enriched BM cells harvested from DKO, p110 $\delta^{-/}$, or WT;Mx1-Cre mice at 3 weeks after 2 pIpC injections, following ex vivo stimulation with IL-1 $\beta$ or TNF- $\alpha$. As expected, we observed a significant decrease in the phosphorylation of AKT at Ser473 in DKO cells, but not in p $110 \delta^{-/-}$cells, with IL-1 $\beta$ or TNF- $\alpha$ stimulation (Figure 6E and Supplemental Figure 8A). This confirmed that p110 $\alpha$ and p110 $\delta$ play redundant roles in the activation of AKT in hematopoietic progenitors. We also observed a trend toward a decrease in phosphorylation of p38 MAPK in DKO cells in response to IL- $1 \beta$ or TNF- $\alpha$, though not to as great an extent as the decrease in AKT phosphorylation in these cells (Figure 6E and Supplemental Figure 8B). Overall, these data suggest that $\mathrm{p} 110 \alpha$ and $\mathrm{p} 110 \delta$ are both required for optimal activation of signaling downstream of IL- $1 \beta$ or TNF- $\alpha$ in hematopoietic stem and progenitor cells in the BM.

To determine whether AKT or p38 MAPK signaling is essential for myelopoiesis after 5-FU administration, we injected WT and DKO mice with $200 \mathrm{mg} / \mathrm{kg} 5-\mathrm{FU}$, and then euthanized the mice after 24 hours, harvested the BM, and performed colony-forming assays in methylcellulose media with myeloid growth factors (M3434, STEMCELL Technologies). We added either the AKT inhibitor MK2206 or the p38 MAPK inhibitor SB203580 to the WT BM cultures. As expected, DKO BM cells formed significantly fewer myeloid colonies than WT; $\mathrm{Cre}^{+}$control BM cells after 5-FU injection (Figure 6F). Interestingly, treatment of WT BM with the AKT inhibitor, but not with the p38 MAPK inhibitor, could phenocopy the results with DKO cells, suggesting that activation of AKT, but not of p38 MAPK, is critical for myeloid differentiation after 5-FU administration (Figure 6F).

p110 $\alpha$ and $p 110 \delta$ are not essential for HSC cell cycle entry in response to LPS administration. To determine whether $\mathrm{p} 110 \alpha$ and $\mathrm{p} 110 \delta$ are also required for the transduction of inflammatory cytokines in HSPCs under conditions simulating bacterial infection, we examined the response of $\mathrm{p} 110 \delta^{-/-}$, DKO, and WT;Mx1-Cre HSPCs to LPS, a bacterial cell wall product (26). LPS is the best-known ligand for TLR signaling and can directly stimulate HSCs in vivo to induce HSC cycling (3). In HSPCs, this process is mediated via the TLR4/TRIF/ROS/p38 pathway and not through MyD88 signaling (3). Upon a single injection of $5 \mu \mathrm{g}$ LPS, HSPCs enter the cell cycle within 24 hours (27). To examine the response of DKO HSPCs to LPS, we treated DKO mice, $\mathrm{p} 110 \delta^{-/-}$littermates, and WT;Mx1-Cre controls with a single $\mathrm{pIpC}$ injection and then administered a single LPS injection after 4 weeks. Mice were sacrificed after 24 hours to examine the cell cycle profile of HSPCs using Hoechst 33342 and Ki-67 staining. Surprisingly, we found that DKO and $\mathrm{p} 110 \delta^{-/-}$HSPCs had cell cycle profiles similar to those of WT;Mx1Cre HSPCs at 24 hours after a single LPS injection (Figure 7A). Consistent with this, we observed phosphorylation of both p38 MAPK and AKT in both WT and DKO HSPCs in response to in vivo LPS administration (Supplemental Figure 8C). Therefore, we conclude that $\mathrm{p} 110 \alpha$ and $\mathrm{p} 110 \delta$ are not required for LPS to activate HSPCs.

Higher levels of inflammatory cytokines and chemokines were detected in the BM after LPS injection than after 5-FU injection. In addition to the direct action of LPS on HSCs via TLR, LPS can also activate HSCs indirectly, by stimulating the secretion of inflammatory cytokines and chemokines by hematopoietic cells and by the BM microenvironment (28). To determine whether differences in cytokine or chemokine levels in the BM could account for some of the differences in HSPC activation that we observed between 5-FU and LPS, we measured the expression of inflammatory cytokines and chemokines in the BM serum at baseline and after injection of 5-FU or LPS using a multiplex Luminex assay. Interestingly, we observed significantly elevated levels of IL-1 $\beta$, IL-1 $\alpha$, and MIG (CXCL9) after exposure to LPS, but not to 5-FU, in WT BM under these conditions (Figure 7B and Supplemental Figure 9A). We also observed significantly increased expression of IL-1 $\beta$ and MIG in $\mathrm{p} 110 \delta^{-/-}$or DKO BM after LPS exposure (Figure 7B). In contrast, 5-FU only increased the expression of MIG in DKO BM, but did not increase the levels of IL-1 $\beta$ in $\mathrm{p} 110 \delta^{-/-}$or DKO BM (Figure 7B). This suggests that LPS but not 5-FU induces increased secretion of some inflammatory cytokines and chemokines in the BM.

DKO HSCs can be activated by in vivo administration of $I L-1 \beta$. It is known that IL-1 $1 \beta$ can have multiple effects in vivo, both cell-autonomous and -nonautonomous. To test the hypothesis that administration of a high dose of IL-1 $\beta$ in vivo can overcome the impaired sensing of IL- $1 \beta$ by DKO HSCs, we injected DKO, p110 $\delta^{-/-}$, or WT;Mx1-Cre mice with $0.5 \mu \mathrm{g}$ IL-1 $\beta$, a dose that we found can induce cycling of WT HSCs in vivo (29) (Supplemental Figure 9B). We then euthanized the mice at 24 hours after injection, harvested the BM, and performed cell cycle analysis with Hoechst and Ki-67. We found that more than 50\% of HSCs from all 3 groups of mice entered the cell cycle in response to IL-1 $\beta$, though there was a trend toward more quiescence 
A

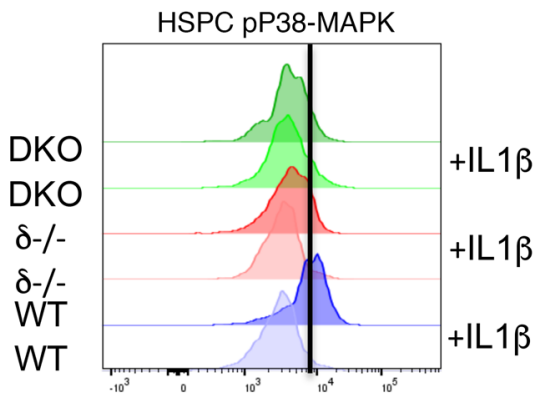

C

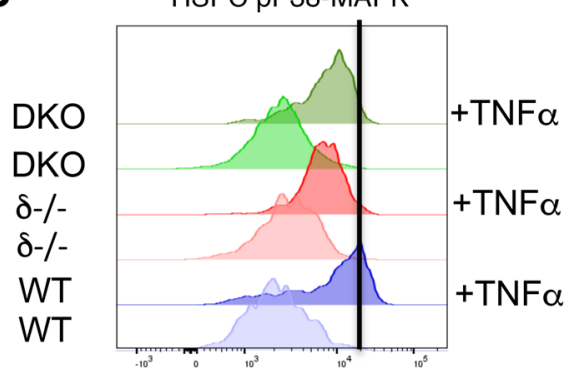

B

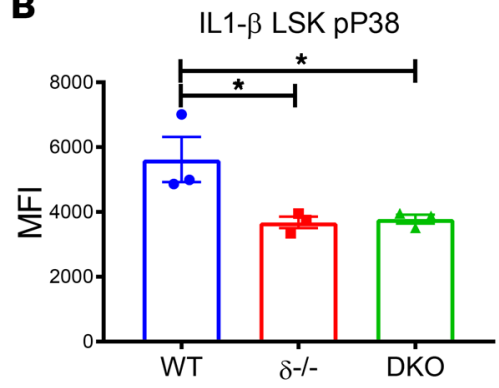

D

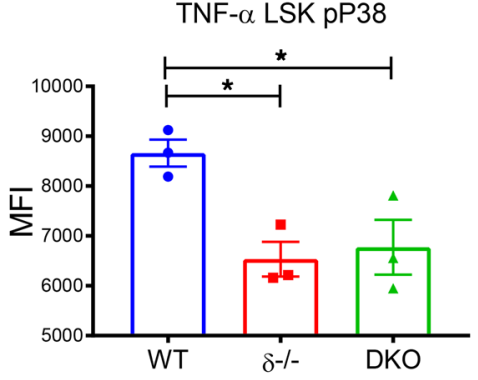

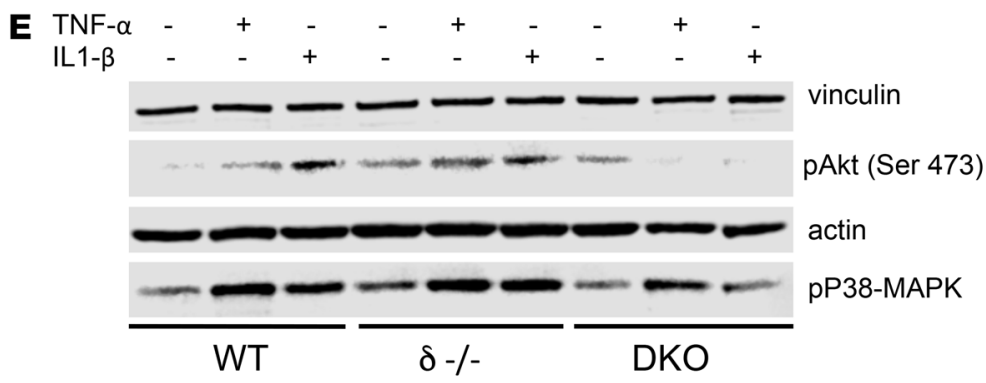

$\mathbf{F}$
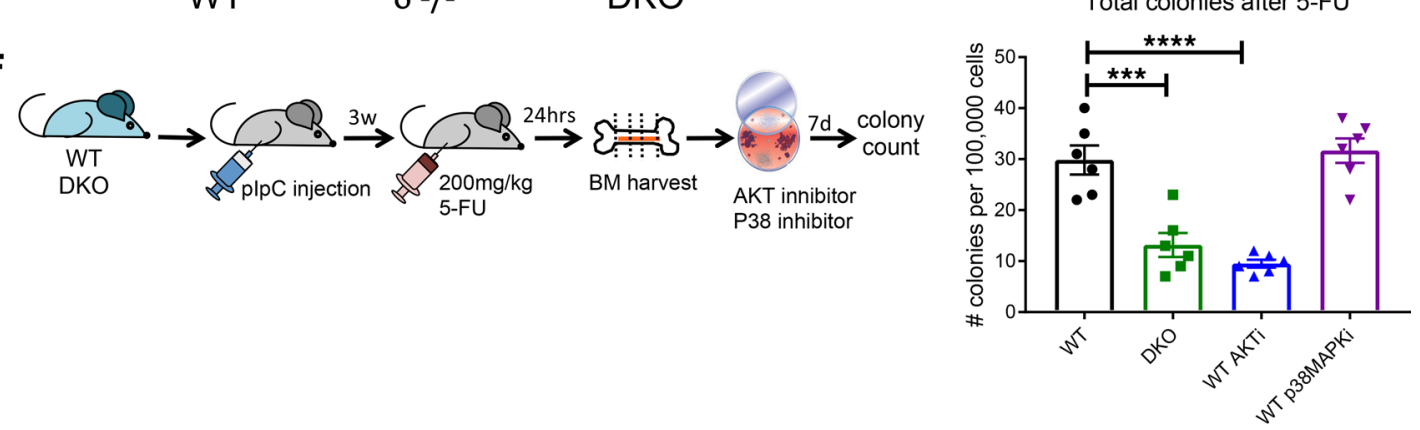

Figure 6. Optimal signal transduction of IL-1 $\beta$ or TNF- $\alpha$ in HSPCs requires $p 110 \alpha$ and $\mathbf{p} 110 \delta$. (A) Phospho-flow cytometry analysis of $p-p 38$ MAPK with IL-1 $\beta$ stimulation in HSPCs (Lin-c-Kit ${ }^{+}$Sca-1+ ${ }^{+}$BM cells). Representative histograms are shown. (B) Quantification of p-p38 median fluorescence intensity (MFI) for the experiment shown in $\mathbf{A}$ (WT; $\mathrm{Cre}, n=3 ; \delta^{-1-}, n=3 ; \mathrm{DKO}, n=3$ ). Data are representative of 3 independent experiments. (C) Phospho-flow cytometry analysis of p-p38 MAPK with TNF- $\alpha$ stimulation in HSPCs. Representative histograms are shown. (D)Quantification of p-p38 MFI for the experiment shown in C (WT;Cre, $n=3 ; \delta^{-1-}, n=3$; DKO, $\left.n=3\right)$. Data are representative of 3 independent experiments. (E) Western blot analysis of c-Kit-enriched BM cells, which were serum starved and then stimulated with IL-1 $\beta$ or TNF- $\alpha$. Representative data from one of 4 independent experiments are shown. Quantification of Western blot data from all experiments is shown in Supplemental Figure 8. (F) BM colony forming assays in methylcellulose media using BM harvested from WT $(n=6)$ or DKO $(n=6)$ mice at 24 hours after 5-FU injection. The AKT inhibitor MK2206 (AKTi; $1 \mu \mathrm{M}$ ) or p-38 MAPK inhibitor SB 203580 (p38MAPKi; $10 \mu \mathrm{M})$ was added to the methylcellulose where indicated. For the experiments in A-D and F, ANOVA with the Tukey's multiple-comparison test was used. ${ }^{*} P<0.05,{ }^{* *} P<0.001,{ }^{* * *} P<0.0001$. Data represent mean \pm SEM.

in the DKO group (Figure 7C). Furthermore, we found that in vivo administration of IL-1 $\beta$ can also induce

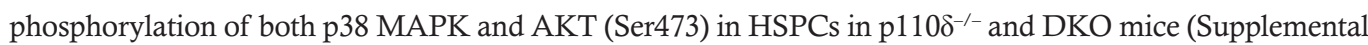
Figure 9 C). This supports the hypothesis that the inefficient transduction of IL-1 $\beta$ signals in DKO HSCs can be at least partially overcome in vivo by high levels of exogenous IL-1 $\beta$. 

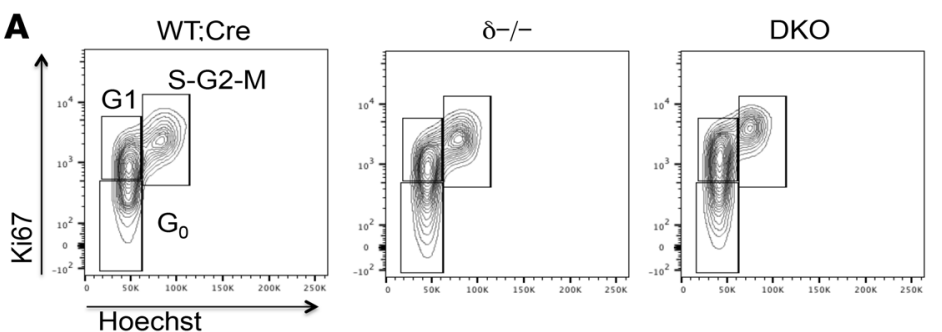

IL $1-\beta$

B
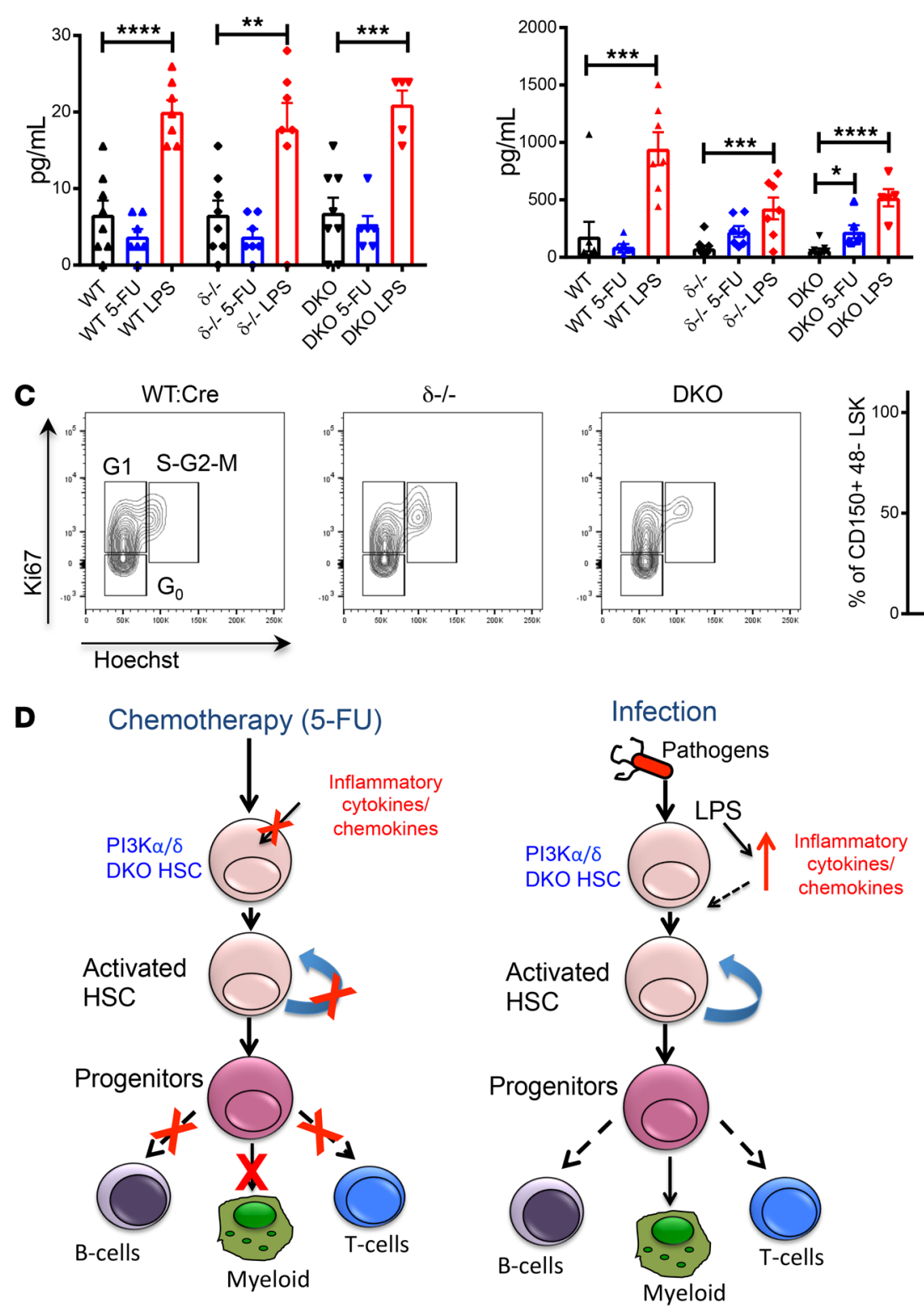

$24 \mathrm{hr}$ post IL1- $\beta$

24hr post LPS
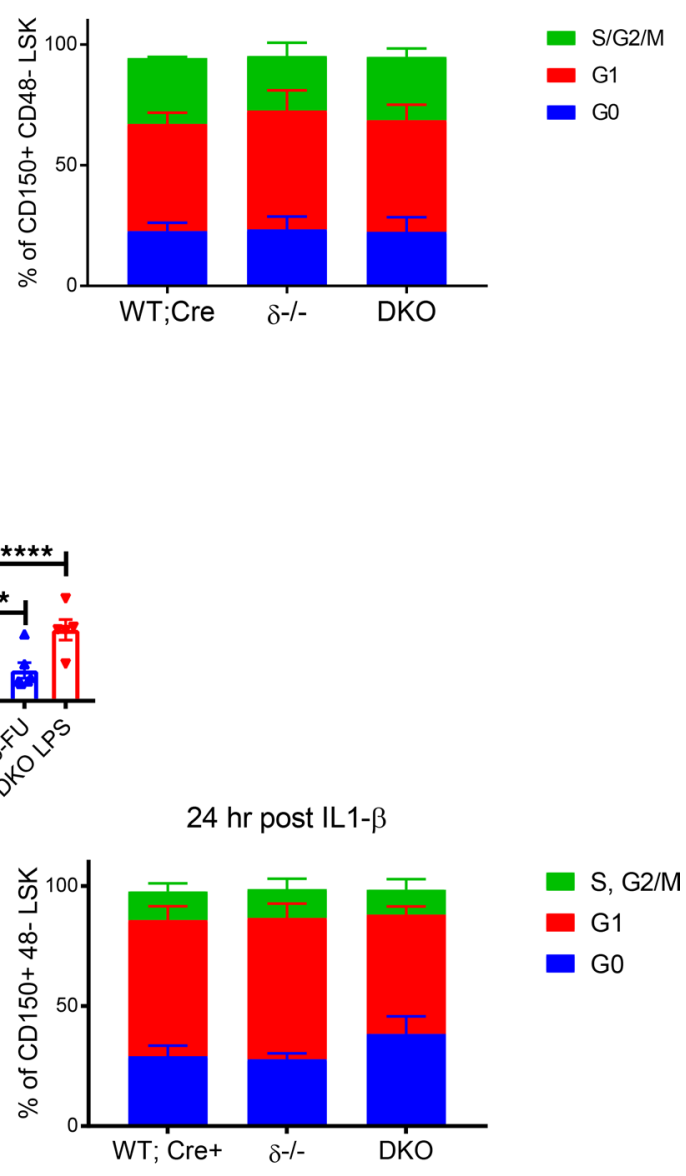

Figure 7. LPS signaling in HSPCs does not require p110 $\alpha$ and p110 $\mathbf{8}$. (A) Representative cell cycle flow cytometry plots of HSCs at 24 hours after a single injection of LPS $(5 \mu \mathrm{g})$. Quantification of the cell cycle analysis is shown on the right. (B) Luminex cytokine assays in BM serum from mice treated with plpC only ( $n=6$ per group), plpC followed by LPS ( $5 \mu \mathrm{g} ; n=6$ per group), or plpC followed by $5-\mathrm{FU}$ (180 mg/kg; $n=6$ per group). (C) Representative cell cycle flow cytometry plots of HSCs at 24 hours after a single i.p. injection of IL-1 $(0.5 \mu \mathrm{g})$. Quantification of the cell cycle analysis is shown on the right. (D) Model figure summarizing the proposed roles of $p 110 \alpha$ and p110 $\delta$ in HSPCs in inflammatory signaling. In the setting of chemotherapy treatment, some inflammatory cytokines and chemokines cannot be efficiently transduced by DKO HSCs, leading to impaired HSC activation and impaired multilineage differentiation. However, increased levels of some inflammatory cytokines or chemokines in the BM after LPS administration can compensate for impaired AKT signal transduction in DKO HSCs and can lead to HSC activation. For the experiments in A-C, ANOVA with the Tukey's multiple-comparisons test was used. ${ }^{*} P<0.05,{ }^{* *} P<0.01,{ }^{* *} P<0.001,{ }^{* * * *} P<0.0001$. Data represent mean $\pm \mathrm{SEM}$. 


\section{Discussion}

It has been reported that $\mathrm{p} 110 \delta^{-/-}$mice have normal blood counts, but the HSC phenotype of these mice has not been previously described (16). Surprisingly, we discovered that while HSCs and progenitors were largely unaffected in $\mathrm{p} 110 \delta^{-/-} \mathrm{BM}$, and $\mathrm{p} 110 \delta^{-/-}$HSC function was normal in competitive repopulation experiments, gene expression was altered in $\mathrm{p} 110 \delta^{-/-}$HSCs and LMPPs. The most pronounced changes we observed in our microarray analysis in $110 \delta^{-/-} \mathrm{HSCs}$ were negative regulation of multiple inflammatory signaling pathways and positive enrichment in $\mathrm{HSC}$-associated genes compared with WT HSCs. In contrast, we observed fewer changes in gene expression in $\mathrm{p} 110 \alpha^{-/-}$HSCs, with the predominant changes reflecting a downregulation in cell cycle-associated gene sets. Only upon deletion of both $\mathrm{p} 110 \alpha$ and $\mathrm{p} 110 \delta$ did we observe a decrease in AKT activation in HSPCs upon IL- $1 \beta$ or TNFstimulation and a significant defect in HSC function under stress. Therefore, our data suggest that $\mathrm{p} 110 \delta$ may play an important role in HSPCs in transducing some inflammatory signals, though p110 $\alpha$ can functionally compensate in $\mathrm{p} 110 \delta^{-/-}$HSCs.

Our studies in $\mathrm{p} 110 \alpha / \delta$-DKO mice suggest that $\mathrm{p} 110 \alpha$ and $\mathrm{p} 110 \delta$ are not critical for HSC function at steady state. The decrease we observed in the numbers of LMPPs and myeloid progenitors in DKO mice, and the competitive repopulation defect that primarily affects the B cell lineage, suggest defects at the level of HSC differentiation or proliferation, as well as in B lineage specification. These findings are consistent with the reported phenotype of compound deletion of the PI3K regulatory isoforms p85 $\alpha$ and p85 $\beta$ in fetal liver HSCs, which were found to be defective in proliferation and in competitive repopulation, particularly in lymphoid lineages (30). However, direct comparisons of adult and fetal HSCs are difficult, given the more proliferative status of fetal HSCs. Using CD2-Cre, which only deletes in B cells, Ramadani et al. have shown that in the absence of activity of both $\mathrm{p} 110 \alpha$ and $\mathrm{p} 110 \delta$, pre-B cell receptor signaling failed to promote developmental progression of B cell progenitors in the BM (21). In our Mx1-Cre system, which allows for deletion of $\mathrm{p} 110 \alpha$ and $\mathrm{p} 110 \delta$ in HSCs and early progenitors, we have uncovered evidence of an earlier defect in B lineage specification. More investigation will be necessary to understand the mechanisms by which $\mathrm{p} 110 \alpha$ and $\mathrm{p} 110 \delta$ promote early fate decisions toward the B cell lineage.

Our results also suggest that additional compensatory mechanisms exist in HSCs that allow for multilineage hematopoietic differentiation and self-renewal at steady state. One possibility is that another PI3K isoform, such as $\mathrm{p} 110 \beta$, could compensate for $\mathrm{p} 110 \alpha$ and $\mathrm{p} 110 \delta$ in HSC self-renewal. Alternatively, a parallel signaling pathway could substitute for PI3K in HSCs. For example, our gene expression analyses suggest the upregulation of the RAS/MAPK signaling pathway in PI3K-KO HSCs, which could be a potential compensatory pathway. This would be consistent with a recent report suggesting that both the MEK/ERK and $\mathrm{AKT} / \mathrm{mMTORC1}$ pathways are activated in HSCs, and that there is feedback regulation between these two pathways in controlling HSC quiescence (31).

In the secondary transplantation recipients, we observed a multilineage reconstitution defect, more severe than the defect that was observed after primary transplantation. Therefore, we conclude that while DKO HSPCs are capable of reconstituting the myeloid lineage at steady state, they are impaired during emergency myelopoiesis. This suggests that while other PI3K isoforms, or parallel signaling pathways, may functionally compensate for $\mathrm{p} 110 \alpha$ and $\mathrm{p} 110 \delta$ in HSCs at steady state, they are critical for HSC self-renewal and differentiation under stress conditions.

Our findings that cell cycle-associated gene sets are significantly downregulated in DKO HSCs are consistent with the known role of the PI3K/AKT pathway in direct regulation of cell cycle intermediates, such as CDK4 $(32,33)$. Surprisingly, we observed significantly decreased survival of DKO mice after a single 5-FU injection, associated with inefficient HSC cell cycle entry. Interestingly, in our previous studies we did not observe any significant defects in hematopoietic recovery in p110 $\alpha$-lox-lox;Mx1-Cre conditional knockout mice after 5-FU administration (12). In addition, we did not observe any significant defect in the survival of $\mathrm{p} 110 \delta^{-/-}$mice after 5 -FU administration, or in the ability of $\mathrm{p} 110 \delta^{-/-}$HSPCs to enter the cell cycle. Therefore, we conclude that $\mathrm{p} 110 \alpha$ and $\mathrm{p} 110 \delta$ play critical but redundant roles in promoting HSPC cell cycle entry under chemotherapy-induced stress.

A recent report has implicated phosphatase and tensin homolog (PTEN) in the inflammatory response in hematopoietic cells (34). Since PTEN antagonizes PI3K signaling, leading to AKT activation, our results are consistent with those of Porter et al., who showed that Pten deletion sensitizes HSCs to growth factors and inflammatory signals. We and others have previously reported on the importance of the PI3K/AKT 
pathway in maintaining the balance between quiescence and proliferation of adult HSCs in studies of AKT pathway activation (35-38). Given the increased cycling of HSCs that was observed in these studies, our observation of increased quiescence in PI3K-KO HSCs is consistent with an important role for PI3K/AKT in controlling HSC quiescence under conditions of hematopoietic stress.

Surprisingly, we observed that DKO HSPCs enter the cell cycle normally upon stimulation with LPS, suggesting that $\mathrm{p} 110 \alpha$ and $\mathrm{p} 110 \delta$ are not absolutely required for HSPC cell cycle entry or progression. Therefore, direct regulation of cell cycle intermediates cannot completely explain the stress hematopoiesis phenotype of DKO mice after 5-FU administration. Our data support the hypothesis that reduced sensing of inflammatory cytokines and chemokines is a potential mechanism for maintained DKO HSC quiescence in response to some forms of hematological stress, such as chemotherapy. Our results suggest that the increased secretion of some inflammatory cytokines and chemokines in the BM upon LPS exposure can overcome the inefficient transduction of such signals by DKO HSPCs, leading to sufficient activation of downstream signaling to induce HSC cycling (Figure 7D). Consistent with this hypothesis, we show that in vivo administration of high-dose exogenous IL-1 $\beta$ can activate HSC cycling and downstream signal transduction in DKO HSCs. It is possible that this effect with in vivo administration of IL-1 $\beta$ is mediated by both cell-autonomous and -nonautonomous functions of IL- $1 \beta$, and may reflect indirect effects of IL-1 $\beta$ on other cell types in the BM. Therefore, the roles of $p 110 \alpha$ and $110 \delta$ in the sensing of specific cytokines or chemokines in HSCs in vivo, and their relative roles in HSC activation, are still unclear. For example, while we observed decreased activation of p38 MAPK and AKT with TNF- $\alpha$ stimulation in DKO HSCs and progenitors, the levels of TNF- $\alpha$ in the BM were not significantly different between these two conditions. Therefore, impaired sensing of TNF- $\alpha$ alone is unlikely to explain the impaired activation of DKO HSCs after 5-FU administration. In addition, it is also possible that direct activation of TLR in DKO HSCs by LPS could play a role in HSC activation. It is likely that proper sensing of multiple inflammatory signals via $\mathrm{p} 110 \alpha$ and $\mathrm{p} 110 \delta$ is required for full HSC activation in response to stress stimuli.

In conclusion, our analysis of $\mathrm{p} 110 \alpha$ and $\mathrm{p} 110 \delta$ deletion in the hematopoietic system has uncovered substantial redundant roles for these two PI3K isoforms in HSC activation in response to hematopoietic stress and in the sensing of some inflammatory signals in HSCs, such as IL-1 $\beta$. Our findings of the importance of the PI3K/AKT pathway in the HSC stress response also have important clinical implications. Several isoform-selective PI3K inhibitors are approved to treat lymphoid malignancies $(39,40)$. For instance, idelalisib, which selectively targets $\mathrm{p} 110 \delta$, is used to treat chronic lymphocytic leukemia (CLL) and indolent non-Hodgkin's lymphoma. However, it has been recently reported that patients treated with idelalisib experience an unexpectedly high rate of neutropenia, especially when it is administered in combination with chemotherapy (41). Copanlisib, a PI3K inhibitor with activity against the p110 $\alpha$ and p110 isoforms, is approved for indolent lymphoma and is being tested in combination with chemotherapy (42). Our results in the DKO mouse model suggest that caution should be exercised when PI3K inhibitors targeting these isoforms are combined with chemotherapy. However, differences in the immune response between mice and humans should also be taken into account when applying these results to the clinic. Clinical trials using alternating sequencing of PI3K inhibitors with chemotherapy should be considered to directly examine the potential for these approaches to minimize toxicity in patients.

\section{Methods}

Mice. Mice were maintained under pathogen-free conditions in barrier facilities at the Dana-Farber Cancer Institute (DFCI) and Albert Einstein College of Medicine (AECOM). Pik3cd germline KO mice (16) were provided as a gift from James Ihle (St. Jude's Children's Hospital, Memphis, Tennessee, USA) as frozen embryos, re-derived by the Boston Children's Hospital Transgenic Facility, and backcrossed to the C57BL/6 strain for a

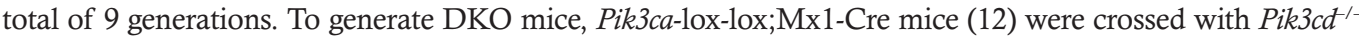
mice, resulting in progeny with the genotypes Pik3ca-lox-lox;Pik3ct ${ }^{-1-}$;Mx1-Cre (DKO) and Pik3ca-lox-lox;Pik$3 c d^{-/-}\left(\mathrm{p} 110 \delta^{-/-}\right)$littermate controls. pIpC (Sigma-Aldrich) was dissolved in HBSS, and $250 \mu \mathrm{g}$ was injected i.p. into 4- to 8-week-old mice twice on nonconsecutive days, unless otherwise indicated. Experimental mice always included both males and females. Peripheral blood was obtained under isoflurane anesthesia by retro-orbital or facial vein bleeding. Genotyping of tail genomic DNA and excision status of exon 1 of $P i k 3 c a$ in the peripheral blood or BM DNA and deletion of Pik3cd were determined by PCR as described previously $(13,16)$. For each experiment, excision of Pik3ca was confirmed in the peripheral blood of DKO mice at 3-4 
weeks after $\mathrm{pIpC}$ injection by peripheral blood analysis. Peripheral blood counts were analyzed on Hemavet (Drew Scientific), Forcyte (Oxford Science), or Genesis (Oxford Science) blood analyzers.

Colony-forming unit assays. Whole BM cells were subjected to RBC lysis (RBC lysis solution, QIAGEN) and resuspended in IMDM media containing 10\% FBS and 1\% penicillin-streptomycin. Cells were seeded at 10,000 cells per dish in methylcellulose semisolid medium (M3434, STEMCELL Technologies). The colonies were scored and phenotyped at 7-10 days after plating.

Colony-forming unit assays after in vivo 5-FU treatment. Whole BM cells were subjected to RBC lysis and resuspended in IMDM media containing 10\% FBS and 1\% penicillin-streptomycin and $1 \mu \mathrm{M} \mathrm{AKT} \mathrm{inhibitor}$ (MK-2206 2HCl, Selleckchem) or $10 \mu \mathrm{M}$ P38 MAPK inhibitor (SB 203580, Selleckchem). The colonies were counted after 7 days of plating.

Flow cytometry. For the flow cytometry analysis of HSPC populations, RBC-lysed BM cells were stained with a panel of lineage and HSC antibodies listed in Supplemental Table 7. Flow cytometry analysis was performed on the BD FACS LSR II. The absolute number of each population per femur was calculated based on the number of whole BM cells flushed from one femur.

Cell cycle analysis. BM cells were first stained with cell surface antibodies (Supplemental Table 7), followed by mild fixation and permeabilization using the FIX \& PERM Cell Fixation \& Permeabilization Kit (Thermo Fisher Scientific) according to the manufacturer's protocol. The fixed and permeabilized cells were incubated with Ki-67-FITC overnight. Hoechst 33342 (Thermo Fisher Scientific) was used to stain DNA at $25 \mu \mathrm{g} / \mathrm{ml}$. Flow cytometric analysis was performed on the BD FACS LSR II.

5-FU treatment. Mice were given a single pIpC injection $(250 \mu \mathrm{g})$ at $4-6$ weeks of age. After 3 weeks, they were injected once i.p. with $200 \mathrm{mg} / \mathrm{kg}$ 5-FU (Sigma-Aldrich). Blood was collected 1 week prior to 5-FU and on days 7, 14, 21, and 28 after 5-FU administration. For cell cycle analysis after 5-FU treatment, mice were injected with $180 \mathrm{mg} / \mathrm{kg}$ i.p. 5-FU at 3 weeks after a single $\mathrm{pIpC}$ injection, and then sacrificed on day 7 after the 5-FU injection.

LPS /IL-1 injection. After a single pIpC injection $(250 \mu \mathrm{g})$ at 4-6 weeks of age, mice were injected once i.p. with $5 \mu \mathrm{g}$ LPS (InvivoGen Ultrapure LPS, E. coli 0111:B4) or $0.5 \mu \mathrm{g}$ IL-1 $\beta$ (PeproTech) and then sacrificed after 24 hours for BM analysis.

Western blotting. BM cells were enriched for c-Kit ${ }^{+}$cells using CD117-bound magnetic beads (Miltenyi Biotec) according to the manufacturer's protocol. The cells were serum starved in IMDM containing $2 \%$ FBS for 1 hour at $37^{\circ} \mathrm{C}$. Cells were stimulated with $10 \mathrm{ng} / \mathrm{mL}$ TNF- $\alpha$ or $100 \mathrm{ng} / \mathrm{mL} \mathrm{IL}-1 \beta$ at $37^{\circ} \mathrm{C}$ for 10 or 20 minutes, respectively. Cells were centrifuged immediately after stimulation, media was aspirated, and the cell pellets were stored at $-80^{\circ} \mathrm{C}$. Cell lysates were prepared in NP-40 low-salt lysis buffer (Boston BioProducts) containing Protease and Phosphatase Inhibitor (Thermo Fisher Scientific), $100 \mu \mathrm{g} / \mathrm{mL}$ PMSF (Roche), and $1 \mathrm{mM}$ sodium orthovanadate (Sigma-Aldrich). Lysates were kept on ice for 30 minutes and then cleared by centrifugation at 14,000 $g$ for 10 minutes at $4^{\circ} \mathrm{C}$. Protein concentration was determined with Bradford assay using Bio-Rad Protein Assay Dye Reagent. Briefly, Western blotting was performed by loading $15 \mu \mathrm{g}$ cell lysate per well on a NuPAGE BisTris $10 \%$ polyacrylamide gel (Thermo Fisher Scientific). Nitrocellulose $0.45-\mu \mathrm{m}$ membrane (Millipore) was used for transfer. Blocking was done in TBST 5\% nonfat milk. The primary antibodies were as follows: phospho-p38 MAPK (Thr180/Tyr182) 1:1000 (catalog 4511S, Cell Signaling Technology), phospho-AKT 1:1000 (catalog4060S, Cell Signaling Technology), vinculin 1:5000 (catalog V9131, Sigma-Aldrich); $\beta$-actin 1:5000 (A2228, Sigma-Aldrich). The secondary antibodies, anti-mouse 680 and anti-rabbit 800 (LI-COR), were used at dilutions of 1:5000 or 1:10,000, respectively. Membranes were developed on the LI-COR Odyssey instrument. Signal quantification and background adjustments were performed using LI-COR Image Studio Lite 5.2 software.

$B M$ and HSC transplantation. For noncompetitive BM transplantation, $1 \times 10^{6}$ whole BM CD45.2 ${ }^{+}$ donor-derived cells from C57BL/ 6 inbred mice were transplanted into lethally irradiated B6.SJL (CD45.1 ${ }^{+}$) mice at 6-8 weeks of age. For lethal irradiation, mice were given a single dose of $950 \mathrm{cGy}$ at least 3 hours prior to transplantation. Donor BM was injected into the retro-orbital plexus. Recipient mice were given Bactrim water for 3 weeks after transplantation. For competitive BM transplantation, $1 \times 10^{6}$ donor-derived whole BM CD 45.2 $2^{+}$cells and $1 \times 10^{6}$ competitor CD45.1 $1^{+} \mathrm{BM}$ cells were transplanted into lethally irradiated B6.SJL mice. After confirmation of engraftment by peripheral blood counts, recipient mice were injected once i.p. with $\mathrm{pIpC}$ at 4 weeks after transplantation. For secondary HSC transplantation, 250 sorted CD $45.2^{+}$LT-HSCs $\left(\mathrm{CD} 150^{+} \mathrm{CD} 48^{-}{ }^{-} \mathrm{Flk}^{-} \mathrm{Lin}^{-} \mathrm{C}-\mathrm{Kit}^{+} \mathrm{Sca}-1^{+}\right)$from the BM of primary noncompetitive transplant recipient mice were mixed with 250,000 WT CD45.1 $1^{+}$CD45.2+ whole BM cells from C57BL/6-B6. SJL $F_{1}$ hybrid mice and transplanted into lethally irradiated 6- to 8-week-old B6.SJL mice. Peripheral blood 
was collected from recipient mice every 4 weeks, and animals were sacrificed after at least 16 weeks of observation after transplantation, or for 16 weeks after $\mathrm{pIpC}$ injection.

Microarray analysis. Lethally irradiated 6- to 8-week-old female B6.SJL mice were transplanted with 2 $\times 10^{6}$ whole BM cells from Pik3ca-lox-lox;Mx1-Cre, Pik3cd;Pik3ca-lox-lox, Pik3cd;Pik3ca-lox-lox;Mx1-Cre, or WT;Mx1-Cre mice ( $n=7$ per group). Peripheral blood was collected at 4 weeks after transplantation to confirm engraftment. All transplant recipients were injected once i.p. with $250 \mu \mathrm{g}$ pIpC. Excision of Pik$3 c a$ was confirmed by examining the blood counts at 3 weeks after $\mathrm{pIpC}$ administration. At 8 weeks after transplantation, BM cells were harvested from femurs, tibiae, ilia, and vertebrae. Bones were gently crushed in RPMI medium (Life Technologies). Low-density BM mononuclear cells (BMMNCs) were isolated by density gradient centrifugation using Ficoll Histopaque-1083 (Sigma-Aldrich). BMMNCs were stained with anti-mouse biotin-labeled lineage antibodies (CD3e, CD4, CD8a, B220, Ter119, CD19, and Gr-1) for 30 minutes at $4^{\circ} \mathrm{C}$. The lineage-stained cells were then incubated with Biotin Binder Dynabeads (Thermo Fisher Scientific, catalog 11047) and depleted with a magnet according to the manufacturer's protocol. To purify HSC and LMPP populations, Lin ${ }^{-}$cells were stained with a panel of fluorochrome-conjugated monoclonal anti-mouse cell surface antibodies (CD45.2-PerCP, CD45.1-FITC, Flk2-PE, IL-7R-PECy7, Sca-1-BV605, c-Kit-APC, CD150-Pacific blue, CD48-Alexa Fluor 700, Streptavidin-APC Cy7; see Supplemental Table 7). The donor-derived HSC and LMPP cell populations were sorted according to the gating strategy in Figure $3 \mathrm{~A}$ using the Aria II instrument (BD Biosciences). Cells were directly sorted into $50 \mu 1$ RNA extraction buffer (ARCTURUS PicoPure RNA Isolation Kit; Life Technologies, Invitrogen) and stored at $-80^{\circ} \mathrm{C}$. RNA isolation was performed using the ARCTURUS PicoPure RNA Isolation Kit according to the manufacturer's protocol. RNA quantification was performed using the RNA Quantification Kit for SYBR Green I and ROX Passive Reference Dye (Thermo Fisher Scientific, catalog 902905). Total RNA was amplified and hybridized to the Gene Chip Mouse Transcriptome Pico Assay 1.0 (Affymetrix, catalog 902663) using the GeneChip Hybridization, Wash, and Stain Kit (catalog 900720). Raw data were analyzed for quality control using Expression Console software (Affymetrix). After quality control, microarray data were analyzed by GSEA using MSigDB software (http://software.broadinstitute.org/gsea/index.jsp), Transcriptome Analysis Software (Affymetrix), Gene ontology enrichment analysis (Gene Ontology Consortium; http:// geneontology.org), and by IPA software (QIAGEN). Venn diagrams were generated using Bioinformatics \& Evolutionary Genomics software (http://bioinformatics.psb.ugent.be/webtools/Venn). The microarray data were deposited in the NCBI's Gene Expression Omnibus database (GEO GSE118053).

Phospho-flow cytometry (after ex vivo stimulation). Mice were injected twice with $\mathrm{pIpC}(250 \mu \mathrm{g})$ at 4-8 weeks of age on nonconsecutive days, and were sacrificed 4-6 weeks after the first pIpC injection. BM cells were harvested and RBC lysis was performed. Phospho-flow cytometry was performed based on Kalaitzidis et al., with the following modifications (43). Cells were stained with a cocktail of biotinylated lineage antibodies (CD127, CD3e, CD4, CD8a, B220, CD19, Ter119, and Gr-1) for 30 minutes. The subsequent depletion of Lin $^{+}$cells was performed using Biotin Binder Dynabeads according to the manufacturer's protocol. Lin ${ }^{-}$cells were serum starved in IMDM containing $2 \%$ FBS for 1 hour at $37^{\circ} \mathrm{C}$. Cells were stimulated with $10 \mathrm{ng}$ TNF- $\alpha$ or $50 \mathrm{ng}$ IL- $1 \beta$ for 10 or 20 minutes, respectively, at $37^{\circ} \mathrm{C}$. Cells were immediately fixed after stimulation with $16 \%$ paraformaldehyde for 10 minutes at room temperature (Electron Microscopy Sciences, catalog 15710). The cells were then centrifuged and permeabilized with cold $100 \%$ acetone for 10 minutes on ice. Fixed cells were stained with a panel of HSPC surface markers (Streptavidin-APC Cy7, c-Kit-PE, Sca-1-BV421) and intracellular phosphop38 MAPK (Thr180/Tyr182)-Alexa Fluor 647 (Cell Signaling Technology, catalog 4552S) antibodies.

Phospho-flow cytometry (after in vivo stimulation). Mice were injected twice with pIpC. At 2-3 weeks after pIpC injection, mice were injected i.p. with $0.5 \mu \mathrm{g}$ IL- $1 \beta$ or $5 \mu \mathrm{g}$ LPS and sacrificed after 24 hours. BM cells were harvested, and RBC lysis was performed. Cells were stained with a cocktail of lineage antibodies as described in the previous section. Cells were stained with a panel of HSPC surface markers (StreptavidinAPC Cy7, c-Kit-PE, Sca-1-PE-Cy7). Cells were then fixed and permeabilized using the FIX \& PERM Cell Fixation \& Cell Permeabilization Kit according to the manufacturer's protocol. The fixed and permeabilized cells were stained with intracellular phospho-p38 MAPK (Thr180/Tyr182)-Alexa Fluor 647 at 3:100 and phospho-AKT (Ser473)-Alexa Fluor 488 (Cell Signaling Technology, catalog 2336S) at 1:20 dilutions.

Cytokine assays. BM sera were collected from mice injected with $\mathrm{pIpC}$ alone, or from mice injected with $\mathrm{pIpC}$ and then once with LPS $(5 \mu \mathrm{g})$ or with $5-\mathrm{FU}(180 \mathrm{mg} / \mathrm{kg})$ at $4-6$ weeks after $\mathrm{pIpC}$ administration. Animals were sacrificed at 24 hours after LPS injection or at 7 days after 5-FU injection. One femur of each mouse was flushed with $200 \mu \mathrm{L}$ RPMI with no additional supplements. The BM cell suspension was centrifuged, and BM 
sera were collected and stored at $-80^{\circ} \mathrm{C}$ for cytokine array analysis. The levels of cytokines in the BM sera were measured using the MILLIPLEX MAP Mouse custom Cytokine/Chemokine Magnetic Bead Panel — Immunology Multiplex Assay (MCYTOMAG-70K-15; EMD Millipore) according to the manufacturer's protocol. The data were acquired using the Luminex 200 instrument.

Microscope image acquisition. Histology images were acquired at room temperature using a Zeiss Axiovert $200 \mathrm{M}$ Microscope with digital camera, using a $\times 10$ (for $\times 100$ images) or $\times 40$ objective (for $\times 400$ imag es). Image acquisition was performed using AxioVision software.

Reagents. Supplemental Table 7 lists the flow cytometry antibodies used. All other reagents are listed in the respective sections in Methods.

Statistics. GraphPad Prism 7 and Microsoft Excel 2010 were used for all statistical analyses. For the comparison of 2 experimental groups, unpaired 2-tailed Student's $t$ test was used. For comparisons of more than 2 groups, ANOVA was used with the Tukey's multiple-comparisons test, unless otherwise indicated. For survival analysis, log-rank analysis was used. The specific statistical test used is also indicated in each figure legend. In all graphs, error bars indicate mean \pm SEM. A $P$ value less than 0.05 was considered significant.

Study approval. All animal experiments were approved by the Institutional Animal Care and Use Committee under protocols 09-069 (DFCI), 20140412 (AECOM), and 20170206 (AECOM).

\section{Author contributions}

$\mathrm{SH}$ and $\mathrm{KG}$ planned and performed experiments, analyzed the data, and wrote the manuscript. TS, MT, ROO, KA, LO, TH, IK, and TSM performed experiments. BB assisted with data analysis. AA performed experiments and assisted with data interpretation. EMP, JJZ, and TMR assisted with the planning of experiments and data interpretation.

\section{Acknowledgments}

We would like to thank all past and current members of the Gritsman laboratory for their helpful suggestions and discussions, and Howard Yan, Linda Clayton, and Dagmar Walter for assistance with experiments. We thank James Ihle and Evan Parganas of St. Jude Children's Research Hospital for providing Pik3cd ${ }^{-1}$ frozen embryos. We thank Daqian Sun of the AECOM Stem Cell Isolation and Xenotransplantation Facility (funded through New York Stem Cell Science grant C029154) and Jinhang Zhang of the AECOM Flow Cytometry Core Facility for assistance with flow cytometry. We thank Peter Schultes of the AECOM Department of Cell Biology. We thank David Reynolds and Wen Tran of the Albert Einstein Genomics facility for assistance with microarray analysis and Britta Will for critical reading of the manuscript. This work was supported by NIH grants R01CA196973 (to KG), K08CA149208 (to KG), R35 CA210057 (to JJZ), R01CA187918 (to TMR and JJZ), and R01DK119394 (to EMP), as well as an American Society of Hematology Scholar Award (to KG) and startup funds from the AECOM and Albert Einstein Cancer Center (to KG). SH was partially supported by the Einstein Training Program in Stem Cell Research from the Empire State Stem Cell Fund through New York State Department of Health contract C30292GG, as well as by Albert Einstein Cancer Center Support grant from the NIH under award number P30CA013330. The content is solely the responsibility of the authors and does not necessarily represent the official views of the NIH.

Address correspondence to: Kira Gritsman, Department of Medicine, Department of Cell Biology, Albert Einstein College of Medicine, New York, New York 10461, USA. Phone: 929.246.6707; Email: kira.gritsman@einstein.yu.edu.

1. Pietras EM. Inflammation: a key regulator of hematopoietic stem cell fate in health and disease. Blood. 2017;130(15):1693-1698.

2. Welner RS, Kincade PW. 9-1-1: HSCs respond to emergency calls. Cell Stem Cell. 2014;14(4):415-416.

3. Takizawa $\mathrm{H}$, et al. Pathogen-induced TLR4-TRIF innate immune signaling in hematopoietic stem cells promotes proliferation but reduces competitive fitness. Cell Stem Cell. 2017;21(2):225-240.e5.

4. Zhao JL, et al. Conversion of danger signals into cytokine signals by hematopoietic stem and progenitor cells for regulation of stress-induced hematopoiesis. Cell Stem Cell. 2014;14(4):445-459.

5. Boettcher S, Manz MG. Regulation of inflammation- and infection-driven hematopoiesis. Trends Immunol. 2017;38(5):345-357.

6. Hemmati S, Haque T, Gritsman K. Inflammatory signaling pathways in preleukemic and leukemic stem cells. Front Oncol. 2017;7:265.

7. Vanhaesebroeck B, Guillermet-Guibert J, Graupera M, Bilanges B. The emerging mechanisms of isoform-specific PI3K signalling. Nat Rev Mol Cell Biol. 2010;11(5):329-341.

8. Sizemore N, Leung S, Stark GR. Activation of phosphatidylinositol 3-kinase in response to interleukin-1 leads to phosphoryla- 
tion and activation of the NF-kappaB p65/RelA subunit. Mol Cell Biol. 1999;19(7):4798-4805.

9. Ozes ON, Mayo LD, Gustin JA, Pfeffer SR, Pfeffer LM, Donner DB. NF-kappaB activation by tumour necrosis factor requires the Akt serine-threonine kinase. Nature. 1999;401(6748):82-85.

10. Hebeis BJ, Vigorito E, Turner M. The p110delta subunit of phosphoinositide 3-kinase is required for the lipopolysaccharide response of mouse B cells. Biochem Soc Trans. 2004;32(pt 5):789-791.

11. Thorpe LM, Yuzugullu H, Zhao JJ. PI3K in cancer: divergent roles of isoforms, modes of activation and therapeutic targeting Nat Rev Cancer. 2015;15(1):7-24.

12. Gritsman K, et al. Hematopoiesis and RAS-driven myeloid leukemia differentially require PI3K isoform p110 $\alpha$. J Clin Invest. 2014;124(4):1794-1809.

13. Zhao JJ, et al. The p110alpha isoform of PI3K is essential for proper growth factor signaling and oncogenic transformation. Proc Natl Acad Sci USA. 2006;103(44):16296-16300.

14. Yuzugullu H, et al. A PI3K p110 $\beta$-Rac signalling loop mediates Pten-loss-induced perturbation of haematopoiesis and leukaemogenesis. Nat Commun. 2015;6:8501

15. Cabezas-Wallscheid N, et al. Identification of regulatory networks in HSCs and their immediate progeny via integrated proteome, transcriptome, and DNA methylome analysis. Cell Stem Cell. 2014;15(4):507-522.

16. Jou ST, et al. Essential, nonredundant role for the phosphoinositide 3-kinase p110delta in signaling by the B-cell receptor complex. Mol Cell Biol. 2002;22(24):8580-8591.

17. Pietras EM, et al. Functionally distinct subsets of lineage-biased multipotent progenitors control blood production in normal and regenerative conditions. Cell Stem Cell. 2015;17(1):35-46.

18. Adolfsson J, et al. Identification of Flt3+ lympho-myeloid stem cells lacking erythro-megakaryocytic potential a revised road map for adult blood lineage commitment. Cell. 2005;121(2):295-306.

19. Hardy RR, Carmack CE, Shinton SA, Kemp JD, Hayakawa K. Resolution and characterization of pro-B and pre-pro-B cell stages in normal mouse bone marrow. J Exp Med. 1991;173(5):1213-1225.

20. Hardy RR, Li YS, Allman D, Asano M, Gui M, Hayakawa K. B-cell commitment, development and selection. Immunol Rev. 2000;175:23-32.

21. Ramadani F, et al. The PI3K isoforms p110alpha and p110delta are essential for pre-B cell receptor signaling and B cell development. Sci Signal. 2010;3(134):ra60.

22. Harrison DE, Lerner CP. Most primitive hematopoietic stem cells are stimulated to cycle rapidly after treatment with 5-fluorouracil. Blood. 1991;78(5):1237-1240.

23. Pietras EM, et al. Chronic interleukin-1 exposure drives haematopoietic stem cells towards precocious myeloid differentiation at the expense of self-renewal. Nat Cell Biol. 2016;18(6):607-618.

24. Karigane D, et al. p38 $\alpha$ Activates purine metabolism to initiate hematopoietic stem/progenitor cell cycling in response to stress. Cell Stem Cell. 2016;19(2):192-204.

25. Gañán-Gómez I, et al. Deregulation of innate immune and inflammatory signaling in myelodysplastic syndromes. Leukemia. 2015;29(7):1458-1469

26. Schuettpelz LG, Link DC. Regulation of hematopoietic stem cell activity by inflammation. Front Immunol. 2013;4:204.

27. Cabezas-Wallscheid N, et al. Vitamin A-retinoic acid signaling regulates hematopoietic stem cell dormancy. Cell. 2017;169(5):807-823.e19.

28. King KY, Goodell MA. Inflammatory modulation of HSCs: viewing the HSC as a foundation for the immune response. Nat Rev Immunol. 2011;11(10):685-692.

29. Weisser M, et al. Hyperinflammation in patients with chronic granulomatous disease leads to impairment of hematopoietic stem cell functions. J Allergy Clin Immunol. 2016;138(1):219-228.e9.

30. Haneline LS, et al. Genetic reduction of class IA PI-3 kinase activity alters fetal hematopoiesis and competitive repopulating ability of hematopoietic stem cells in vivo. Blood. 2006;107(4):1375-1382.

31. Baumgartner C, et al. An ERK-dependent feedback mechanism prevents hematopoietic stem cell exhaustion. Cell Stem Cell. 2018;22(6):879-892.e6.

32. Pietras EM, Warr MR, Passegue E. Cell cycle regulation in hematopoietic stem cells. J Cell Biol. 2011;195(5):709-720.

33. Liang J, Slingerland JM. Multiple roles of the PI3K/PKB (Akt) pathway in cell cycle progression. Cell Cycle. 2003;2(4):339-345.

34. Porter SN, et al. Pten cell autonomously modulates the hematopoietic stem cell response to inflammatory cytokines. Stem Cell Reports. 2016;6(6):806-814.

35. Kharas MG, et al. Constitutively active AKT depletes hematopoietic stem cells and induces leukemia in mice. Blood. 2010;115(7):1406-1415.

36. Magee JA, Ikenoue T, Nakada D, Lee JY, Guan KL, Morrison SJ. Temporal changes in PTEN and mTORC2 regulation of hematopoietic stem cell self-renewal and leukemia suppression. Cell Stem Cell. 2012;11(3):415-428.

37. Yilmaz $\mathrm{OH}$, et al. Pten dependence distinguishes haematopoietic stem cells from leukaemia-initiating cells. Nature. 2006;441(7092):475-482.

38. Zhang J, et al. PTEN maintains haematopoietic stem cells and acts in lineage choice and leukaemia prevention. Nature. 2006;441(7092):518-522.

39. Lampson BL, Brown JR. PI3K $\delta$-selective and PI3K $\alpha / \delta$-combinatorial inhibitors in clinical development for B-cell non-Hodgkin lymphoma. Expert Opin Investig Drugs. 2017;26(11):1267-1279.

40. Fruman DA, Rommel C. PI3Kdelta inhibitors in cancer: rationale and serendipity merge in the clinic. Cancer Discov. 2011;1(7):562-572.

41. Zelenetz $\mathrm{AD}$, et al. Idelalisib or placebo in combination with bendamustine and rituximab in patients with relapsed or refractory chronic lymphocytic leukaemia: interim results from a phase 3, randomised, double-blind, placebo-controlled trial. Lancet Oncol. 2017;18(3):297-311.

42. Markham A. Copanlisib: first global approval. Drugs. 2017;77(18):2057-2062.

43. Kalaitzidis D, Neel BG. Flow-cytometric phosphoprotein analysis reveals agonist and temporal differences in responses of murine hematopoietic stem/progenitor cells. PLoS ONE. 2008;3(11):e3776. 